\title{
KMS STATES AND COMPLEX MULTIPLICATION (PART II)
}

\author{
ALAIN CONNES, MATILDE MARCOLLI, AND NIRANJAN RAMACHANDRAN
}

\section{Contents}

1. Introduction 1

1.1. Notation 3

2. Quantum Statistical Mechanics and Explicit Class Field Theory 4

2.1. Quantum Statistical Mechanics 4

2.2. Hilbert's 12th problem 6

2.3. Fabulous states for number fields 6

2.4. Noncommutative pro-varieties $\quad 7$

3. $\mathbb{Q}$-lattices and noncommutative Shimura varieties 8

3.1. Tower Power 9

3.2. The cyclotomic tower and the BC system 9

3.3. Arithmetic structure of the BC system 11

3.4. The modular tower and the $\mathrm{GL}_{2}$-system 14

3.5. Tate modules and Shimura varieties 16

3.6. Arithmetic properties of the $\mathrm{GL}_{2}$-system 18

3.7. Crossed products and functoriality 20

4. Quantum statistical mechanics for imaginary quadratic fields 22

4.1. $K$-lattices and commensurability 23

4.2. Algebras of coordinates 25

4.3. Symmetries 27

4.4. Comparison with other systems 28

5. KMS states and complex multiplication 28

5.1. Low temperature KMS states and Galois action 29

5.2. Open Questions 31

References $\quad 31$

\section{INTRODUCTION}

Several results point to deep relations between noncommutative geometry and class field theory ([3], [10], [20], [22]). In [3] a quantum statistical mechanical system (BC) is exhibited, with partition function the Riemann zeta function $\zeta(\beta)$, and whose arithmetic properties are related to the Galois theory of the maximal abelian extension of $\mathbb{Q}$. In $[10]$, this system is reinterpreted in terms of the geometry of commensurable 1-dimensional $\mathbb{Q}$-lattices, and a generalization is constructed for 2-dimensional $\mathbb{Q}$-lattices. The arithmetic properties of this $\mathrm{GL}_{2}$-system and its extremal KMS states at zero temperature are related to the Galois theory of the modular field $F$, that is, the field of elliptic modular functions. These are functions on modular curves, i.e. on moduli spaces of elliptic curves. The low temperature extremal KMS states and the Galois properties of the $\mathrm{GL}_{2}$-system are analyzed in [10] for the generic case of elliptic curves with transcendental $j$-invariant. As the results of [10] show, one of the main new features of the $\mathrm{GL}_{2}$-system is the presence of symmetries by endomorphism, as in (2.6) below. The full Galois group of the modular field appears then as symmetries, acting on the set of extremal $\mathrm{KMS}_{\beta}$ states of the system, for large inverse temperature $\beta$. 
In both the original $\mathrm{BC}$ system and in the $\mathrm{GL}_{2}$-system, the arithmetic properties of zero temperature KMS states rely on an underlying result of compatibility between adèlic groups of symmetries and Galois groups. This correspondence between adèlic and Galois groups naturally arises within the context of Shimura varieties. In fact, a Shimura variety is a pro-variety defined over $\mathbb{Q}$, with a rich adèlic group of symmetries. In that context, the compatibility of the Galois action and the automorphisms is at the heart of Langlands program. This leads us to give a reinterpretation of the $\mathrm{BC}$ and the $\mathrm{GL}_{2}$ systems in the language of Shimura varieties, with the $\mathrm{BC}$ system corresponding to the simplest (zero dimensional) Shimura variety $\operatorname{Sh}\left(\mathrm{GL}_{1}, \pm 1\right.$ ). In the case of the $\mathrm{GL}_{2}$ system, we show how the data of 2 -dimensional $\mathbb{Q}$-lattices and commensurability can be also described in terms of elliptic curves together with a pair of points in the total Tate module, and the system is related to the Shimura variety $\operatorname{Sh}\left(\mathrm{GL}_{2}, \mathbb{H}^{ \pm}\right)$of $\mathrm{GL}_{2}$. This viewpoint suggests considering our systems as noncommutative pro-varieties defined over $\mathbb{Q}$, more specifically as noncommutative Shimura varieties.

This point of view, which we discuss here only in the simplest case of $G=\mathrm{GL}_{1}$ and $G=\mathrm{GL}_{2}$ was extended to a wide class of Shimura varieties by E. Ha and F. Paugam, [13]. They constructed generalizations of the $\mathrm{BC}$ and $\mathrm{GL}_{2}$ system that provide noncommutative Shimura varieties, and investigated the arithmetic properties of their partition functions and KMS states.

In our paper [11], we construct a quantum statistical mechanical system associated to an imaginary quadratic field $K=\mathbb{Q}(\sqrt{-d}), d>0$ a positive integer. Just like the $\mathrm{BC}$ and the $\mathrm{GL}_{2}$ systems are based on the geometric notion of $\mathbb{Q}$-lattices and commensurability, this "complex multiplication system" (CM) is based on an analogous geometric notion of commensurability of 1-dimensional $K$ lattices.

The arithmetic properties of the CM system fully incorporate the explicit class field theory for the imaginary quadratic field $K$, and its partition function is the Dedekind zeta function $\zeta_{K}(\beta)$ of $K$. Thus, the main result of [11], which we recall here in Theorem 5.2 below, gives a complete answer, in the case of an imaginary quadratic field $K$, to the following question, which has been open since the work of Bost and Connes [3].

Problem 1.1. For some number field $K$ (other than $\mathbb{Q}$ ) exhibit an explicit quantum statistical mechanical system $\left(\mathcal{A}, \sigma_{t}\right)$ with the following properties:

(1) The partition function $Z(\beta)$ is the Dedekind zeta function of $K$.

(2) The system has a phase transition with spontaneous symmetry breaking at the pole $\beta=1$ of the zeta function.

(3) There is a unique equilibrium state above critical temperature.

(4) The quotient $C_{K} / D_{K}$ of the idèles class group of $K$ by the connected component of the identity acts as symmetries of the system $\left(\mathcal{A}, \sigma_{t}\right)$.

(5) There is a subalgebra $\mathcal{A}_{0}$ of $\mathcal{A}$ with the property that the values of extremal ground states on elements of $\mathcal{A}_{0}$ are algebraic numbers and generate the maximal abelian extension $K^{a b}$.

(6) The Galois action on these values is realized by the induced action of $C_{K} / D_{K}$ on the ground states, via the class field theory isomorphism $\theta: C_{K} / D_{K} \rightarrow \operatorname{Gal}\left(K^{a b} / K\right)$.

The BC system satisfies all the properties listed in Problem 1.1, in the case of $K=\mathbb{Q}$. It is natural, therefore, to pose the analogous question in the case of other number fields. Some important progress in the direction of generalizing the BC system to other number fields was done by Harari and Leichtnam [16], Cohen [5], Arledge, Laca and Raeburn [1], Laca and van Frankenhuijsen [20]. However, to our knowledge, the first construction of a system satisfying all the conditions posed in Problem 1.1, with no restriction on the class number of $K$, was the one obtained in [11]. As we shall discuss at length in the present paper, one reason why progress in the solution of Problem 1.1 is difficult is that the requirement on the Galois action on ground states (zero temperature KMS states) of the system is very closely related to a difficult problem in number theory, namely Hilbert's 12 th problem on explicit class field theory. We will argue in this paper that Problem 1.1 may provide a new possible approach to Hilbert's 12th problem. 
In this perspective, the BC system and the CM system of [11] cover the two known cases $(K=\mathbb{Q}$ and $K=\mathbb{Q}(\sqrt{-d})$ ) of Hilbert's 12th problem. We analyze closely the properties of both the BC and the CM system, in order to understand what new insight they may give on this problem and in what part instead they depend on the number theoretic solution.

The new CM system we constructed in [11] can be regarded in two different ways. On the one hand, it is a generalization of the BC system of [3], when changing the field from $\mathbb{Q}$ to $K=\mathbb{Q}(\sqrt{-d})$, and is in fact Morita equivalent to the one considered in [20], but with no restriction on the class number. On the other hand, it is also a specialization of the $\mathrm{GL}_{2}$-system of [10] to elliptic curves with complex multiplication by $K$. The $\mathrm{KMS}_{\infty}$ states of the CM system can be related to the non-generic $\mathrm{KMS}_{\infty}$ states of the $\mathrm{GL}_{2}$-system, associated to points $\tau \in \mathbb{H}$ with complex multiplication by $K$, and the group of symmetries is the Galois group of the maximal abelian extension of $K$.

Here also symmetries by endomorphisms play a crucial role, as they allow for the action of the class group $\mathrm{Cl}(\mathcal{O})$ of the ring $\mathcal{O}$ of algebraic integers of $K$, so that the properties of Problem 1.1 are satisfied in all cases, with no restriction on the class number of $K$.

As we showed in [11], the CM system can be realized as a subgroupoid of the $\mathrm{GL}_{2}$-system. It has then a natural choice of an arithmetic subalgebra inherited from that of the $\mathrm{GL}_{2}$-system. This is crucial, in order to obtain the intertwining of Galois action on the values of extremal KMS states and action of symmetries of the system.

The paper is structured as follows. In Sections 2 and 3 we discuss the relation between Problem 1.1 and Hilbert's 12th problem and the geometry of the $\mathrm{BC}$ and $\mathrm{GL}_{2}$ system from the point of view of Shimura varieties. In Section 4 we recall the construction and main properties of the CM system, especially its relation to the explicit class field theory for imaginary quadratic fields. We also compare it with the $\mathrm{BC}$ and $\mathrm{GL}_{2}$ systems and with previous systems introduced as generalizations of the $\mathrm{BC}$ system.

We summarize and compare the main properties of the three systems $\left(\mathrm{BC}, \mathrm{GL}_{2}\right.$, and $\left.\mathrm{CM}\right)$ in the following table.

\begin{tabular}{|c|c|c|c|}
\hline System & $\mathrm{GL}_{1}$ & $\mathrm{GL}_{2}$ & $\mathrm{CM}$ \\
\hline Partition function & $\zeta(\beta)$ & $\zeta(\beta) \zeta(\beta-1)$ & $\zeta_{K}(\beta)$ \\
\hline Symmetries & $\mathbb{A}_{f}^{*} / \mathbb{Q}^{*}$ & $\mathrm{GL}_{2}\left(\mathbb{A}_{f}\right) / \mathbb{Q}^{*}$ & $\mathbb{A}_{K, f}^{*} / K^{*}$ \\
\hline Symmetry group & Compact & Locally compact & Compact \\
\hline Automorphisms & $\hat{\mathbb{Z}}^{*}$ & $\mathrm{GL}_{2}(\hat{\mathbb{Z}})$ & $\hat{\mathcal{O}}^{*} / \mathcal{O}^{*}$ \\
\hline Endomorphisms & & $\mathrm{GL}_{2}^{+}(\mathbb{Q})$ & $\mathrm{Cl}(\mathcal{O})$ \\
\hline Galois group & $\mathrm{Gal}\left(\mathbb{Q}^{a b} / \mathbb{Q}\right)$ & $\mathrm{Aut}(F)$ & $\mathrm{Gal}\left(K^{a b} / K\right)$ \\
\hline Extremal KMS & $S h\left(\mathrm{GL}_{1}, \pm 1\right)$ & $S h\left(\mathrm{GL}_{2}, \mathbb{H}^{ \pm}\right)$ & $\mathbb{A}_{K, f}^{*} / K^{*}$ \\
\hline
\end{tabular}

\subsection{Notation.}

In the table above and in the rest of the paper, we denote by $\hat{\mathbb{Z}}$ the profinite completion of $\mathbb{Z}$ and by $\mathbb{A}_{f}=\hat{\mathbb{Z}} \otimes \mathbb{Q}$ the ring of finite adeles of $\mathbb{Q}$. For any abelian group $G$, we denote by $G_{\text {tors }}$ the subgroup of elements of finite order. For any ring $R$, we write $R^{*}$ for the group of invertible elements, while $R^{\times}$denotes the set of nonzero elements of $R$, which is a semigroup if $R$ is an integral domain. We 
write $\mathcal{O}$ for the ring of algebraic integers of the imaginary quadratic field $K=\mathbb{Q}(\sqrt{-d})$, where $d$ is a positive integer. We also use the notation

$$
\hat{\mathcal{O}}:=(\mathcal{O} \otimes \hat{\mathbb{Z}}) \quad \mathbb{A}_{K, f}=\mathbb{A}_{f} \otimes_{\mathbb{Q}} K \quad \text { and } \quad \mathbb{I}_{K}=\mathbb{A}_{K, f}^{*}=\operatorname{GL}_{1}\left(\mathbb{A}_{K, f}\right) .
$$

Notice that $K^{*}$ embeds diagonally into $\mathbb{I}_{K}$.

The modular field $F$ is the field of modular functions over $\mathbb{Q}^{a b}$ (cf. e.g. [21]). This is the union of the fields $F_{N}$ of modular functions of level $N$ rational over the cyclotomic field $\mathbb{Q}\left(\zeta_{N}\right)$, that is, such that the $q$-expansion at a cusp has coefficients in the cyclotomic field $\mathbb{Q}\left(\zeta_{N}\right)$.

G. Shimura determined the automorphisms of $F(c f$. [32]). His result

$$
\mathrm{GL}_{2}\left(\mathbb{A}_{f}\right) / \mathbb{Q}^{*} \stackrel{\sim}{\longrightarrow} \operatorname{Aut}(F),
$$

is a non-commutative analogue of the class field theory isomorphism which provides the canonical identifications

$$
\theta: \mathbb{I}_{K} / K^{*} \stackrel{\sim}{\longrightarrow} \operatorname{Gal}\left(K^{a b} / K\right)
$$

and $\mathbb{A}_{f}^{*} / \mathbb{Q}_{+}^{*} \stackrel{\sim}{\longrightarrow} \operatorname{Gal}\left(\mathbb{Q}^{a b} / \mathbb{Q}\right)$.

\section{Quantum Statistical Mechanics and Explicit Class Field Theory}

The BC quantum statistical mechanical system [2,3] exhibits generators of the maximal abelian extension of $\mathbb{Q}$, parameterizing extremal zero temperature states. Moreover, the system has the remarkable property that extremal $\mathrm{KMS}_{\infty}$ states take algebraic values, when evaluated on a rational subalgebra of the $C^{*}$-algebra of observables. The action on these values of the absolute Galois group factors through the abelianization $\operatorname{Gal}\left(\mathbb{Q}^{a b} / \mathbb{Q}\right)$ and is implemented by the action of the idèle class group as symmetries of the system, via the class field theory isomorphism. This suggests the intriguing possibility of using the setting of quantum statistical mechanics to address the problem of explicit class field theory for other number fields.

In this section we recall some basic notions of quantum statistical mechanics and of class field theory, which will be used throughout the paper. We also formulate a general conjectural relation between quantum statistical mechanics and the explicit class field theory problem for number fields.

\subsection{Quantum Statistical Mechanics.}

A quantum statistical mechanical system consists of an algebra of observables, given by a unital $C^{*}$ algebra $\mathcal{A}$, together with a time evolution, consisting of a 1-parameter group of automorphisms $\sigma_{t}$, $(t \in \mathbb{R})$, whose infinitesimal generator is the Hamiltonian of the system, $\sigma_{t}(x)=e^{i t H} x e^{-i t H}$. The analog of a probability measure, assigning to every observable a certain average, is given by a state, namely a continuous linear functional $\varphi: \mathcal{A} \rightarrow \mathbb{C}$ satisfying positivity, $\varphi\left(x^{*} x\right) \geq 0$, for all $x \in \mathcal{A}$, and normalization, $\varphi(1)=1$. In the quantum mechanical framework, the analog of the classical Gibbs measure is given by states satisfying the KMS condition ( $c f .[15])$.

Definition 2.1. A triple $\left(\mathcal{A}, \sigma_{t}, \varphi\right)$ satisfies the Kubo-Martin-Schwinger (KMS) condition at inverse temperature $0 \leq \beta<\infty$, if the following holds. For all $x, y \in \mathcal{A}$, there exists a holomorphic function $F_{x, y}(z)$ on the strip $0<\operatorname{Im}(z)<\beta$, which extends as a continuous function on the boundary of the strip, with the property that

$$
F_{x, y}(t)=\varphi\left(x \sigma_{t}(y)\right) \quad \text { and } \quad F_{x, y}(t+i \beta)=\varphi\left(\sigma_{t}(y) x\right), \quad \forall t \in \mathbb{R} .
$$

We also say that $\varphi$ is a $\mathrm{KMS}_{\beta}$ state for $\left(\mathcal{A}, \sigma_{t}\right)$. The set $\mathcal{K}_{\beta}$ of $\mathrm{KMS}_{\beta}$ states is a compact convex Choquet simplex [4, II $\S 5]$ whose set of extreme points $\mathcal{E}_{\beta}$ consists of the factor states. One can express any $\mathrm{KMS}_{\beta}$ state uniquely in terms of extremal states, because of the uniqueness of the barycentric decomposition of a Choquet simplex. 
At 0 temperature $(\beta=\infty)$ the KMS condition (2.1) says that, for all $x, y \in \mathcal{A}$, the function

$$
F_{x, y}(t)=\varphi\left(x \sigma_{t}(y)\right)
$$

extends to a bounded holomorphic function in the upper half plane $\mathbb{H}$. This implies that, in the Hilbert space of the GNS representation of $\varphi$ (i.e. the completion of $\mathcal{A}$ in the inner product $\varphi\left(x^{*} y\right)$ ), the generator $H$ of the one-parameter group $\sigma_{t}$ is a positive operator (positive energy condition). However, this notion of 0 -temperature KMS states is in general too weak, hence the notion of $\mathrm{KMS}_{\infty}$ states that we shall consider is the following.

Definition 2.2. A state $\varphi$ is a $K M S_{\infty}$ state for $\left(\mathcal{A}, \sigma_{t}\right)$ if it is a weak limit of $K M S_{\beta}$ states, that is, $\varphi_{\infty}(a)=\lim _{\beta \rightarrow \infty} \varphi_{\beta}(a)$ for all $a \in \mathcal{A}$.

One can easily see the difference between the KMS condition at zero tempretature and the notion of $\mathrm{KMS}_{\infty}$ states given in Definition 2.2, in the simple case of the trivial time evolution $\sigma_{t}=\mathrm{id}, \quad \forall t \in \mathbb{R}$. In this case, any state has the property that (2.2) extends to the upper half plane (as a constant). On the other hand, only tracial states can be weak limits of $\beta$-KMS states, hence the notion given in Definition 2.2 is more restrictive. With Definition 2.2 we still obtain a weakly compact convex set $\Sigma_{\infty}$ and we can consider the set $\mathcal{E}_{\infty}$ of its extremal points.

The typical framework for spontaneous symmetry breaking in a system with a unique phase transition (cf. [14]) is that the simplex $\Sigma_{\beta}$ consists of a single point for $\beta \leqq \beta_{c}$ i.e. when the temperature is larger than the critical temperature $T_{c}$, and is non-trivial (of some higher dimension in general) when the temperature lowers. A (compact) group of automorphisms $G \subset \operatorname{Aut}(\mathcal{A})$ commuting with the time evolution,

$$
\sigma_{t} \alpha_{g}=\alpha_{g} \sigma_{t} \quad \forall g \in G, t \in \mathbb{R},
$$

is a symmetry group of the system. Such $G$ acts on $\Sigma_{\beta}$ for any $\beta$, hence on the set of extreme points $\mathcal{E}\left(\Sigma_{\beta}\right)=\mathcal{E}_{\beta}$. The choice of an equilibrium state $\varphi \in \mathcal{E}_{\beta}$ may break this symmetry to a smaller subgroup given by the isotropy group $G_{\varphi}=\{g \in G, g \varphi=\varphi\}$.

The unitary group $\mathcal{U}$ of the fixed point algebra of $\sigma_{t}$ acts by inner automorphisms of the dynamical system $\left(\mathcal{A}, \sigma_{t}\right)$, by

$$
(\operatorname{Ad} u)(a):=u a u^{*}, \quad \forall a \in \mathcal{A},
$$

for all $u \in \mathcal{U}$. One can define an action modulo inner of a group $G$ on the system $\left(\mathcal{A}, \sigma_{t}\right)$ as a map $\alpha: G \rightarrow \operatorname{Aut}\left(\mathcal{A}, \sigma_{t}\right)$ fulfilling the condition

$$
\alpha(g h) \alpha(h)^{-1} \alpha(g)^{-1} \in \operatorname{Inn}\left(\mathcal{A}, \sigma_{t}\right), \quad \forall g, h \in G,
$$

i.e. , as a homomorphism $\alpha: G \rightarrow \operatorname{Aut}\left(A, \sigma_{t}\right) / \mathcal{U}$. The $\mathrm{KMS}_{\beta}$ condition shows that the inner automorphisms $\operatorname{Inn}\left(\mathcal{A}, \sigma_{t}\right)$ act trivially on $\mathrm{KMS}_{\beta}$ states, hence (2.5) induces an action of the group $G$ on the set $\Sigma_{\beta}$ of $\mathrm{KMS}_{\beta}$ states, for $0<\beta \leq \infty$.

More generally, one can consider actions by endomorphisms (cf. [10]), where an endomorphism $\rho$ of the dynamical system $\left(\mathcal{A}, \sigma_{t}\right)$ is a $*$-homomorphism $\rho: \mathcal{A} \rightarrow \mathcal{A}$ commuting with the evolution $\sigma_{t}$. There is an induced action of $\rho$ on $\mathrm{KMS}_{\beta}$ states, for $0<\beta<\infty$, given by

$$
\rho^{*}(\varphi):=\frac{\varphi \circ \rho}{\varphi(\rho(1))}
$$

provided that $\varphi(\rho(1)) \neq 0$, where $\rho(1)$ is an idempotent fixed by $\sigma_{t}$.

An isometry $u \in \mathcal{A}, u^{*} u=1$, satisfying $\sigma_{t}(u)=\lambda^{i t} u$ for all $t \in \mathbb{R}$ and for some $\lambda \in \mathbb{R}_{+}^{*}$, defines an inner endomorphism $\operatorname{Ad} u$ of the dynamical system $\left(\mathcal{A}, \sigma_{t}\right)$, again of the form (2.4). The $\mathrm{KMS}_{\beta}$ condition shows that the induced action of $\operatorname{Ad} u$ on $\Sigma_{\beta}$ is trivial, $c f$. [10].

In general, the induced action (modulo inner) of a semigroup of endomorphisms of $\left(\mathcal{A}, \sigma_{t}\right)$ on the $\mathrm{KMS}_{\beta}$ states need not extend directly (in a nontrivial way) to $\mathrm{KMS}_{\infty}$ states. In fact, even though (2.6) is defined for states $\varphi_{\beta} \in \mathcal{E}_{\beta}$, it can happen that, when passing to the weak $\operatorname{limit} \varphi=\lim _{\beta} \varphi_{\beta}$ one has $\varphi(\rho(1))=0$ and can no longer apply (2.6). 
In such cases, it is often still possible to obtain an induced nontrivial action on $\mathcal{E}_{\infty}$. This can be done via the following procedure, which was named "warming up and cooling down" in [10]. One considers first a map $W_{\beta}: \mathcal{E}_{\infty} \rightarrow \mathcal{E}_{\beta}$ (called the "warming up" map) given by

$$
W_{\beta}(\varphi)(a)=\frac{\operatorname{Tr}\left(\pi_{\varphi}(a) e^{-\beta H}\right)}{\operatorname{Tr}\left(e^{-\beta H}\right)}, \quad \forall a \in \mathcal{A} .
$$

Here $H$ is the positive energy Hamiltonian, implementing the time evolution in the GNS representation $\pi_{\varphi}$ associated to the extremal $\mathrm{KMS}_{\infty}$ state $\varphi$. Assume that, for sufficiently large $\beta$, the map (2.7) gives a bijection between $\mathrm{KMS}_{\infty}$ states (in the sense of Definition 2.2) and $\mathrm{KMS}_{\beta}$ states. The action by endomorphisms on $\mathcal{E}_{\infty}$ is then defined as

$$
\rho^{*}(\varphi)(a):=\lim _{\beta \rightarrow \infty} \rho^{*}\left(W_{\beta}(\varphi)\right)(a) \quad \forall a \in \mathcal{A} .
$$

This type of symmetries, implemented by endomorphisms instead of automorphisms, plays a crucial role in the theory of superselection sectors in quantum field theory, developed by Doplicher-HaagRoberts (cf.[14], Chapter IV).

States on a $C^{*}$-algebra extend the notion of integration with respect to a measure in the commutative case. In the case of a non-unital algebra, the multipliers algebra provides a compactification, which corresponds to the Stone-Cech compactification in the commutative case. A state admits a canonical extension to the multiplier algebra. Moreover, just as in the commutative case one can extend integration to certain classes of unbounded functions, it is preferable to extend, whenever possible, the integration provided by a state to certain classes of unbounded multipliers.

\subsection{Hilbert's 12th problem.}

The main theorem of class field theory provides a classification of finite abelian extensions of a local or global field $K$ in terms of subgroups of a locally compact abelian group canonically associated to the field. This is the multiplicative group $K^{*}=\mathrm{GL}_{1}(K)$ in the local non-archimedean case, while in the global case it is the quotient of the idèle class group $C_{K}$ by the connected component of the identity. The construction of the group $C_{K}$ is at the origin of the theory of idèles and adèles.

Hilbert's 12th problem can be formulated as the question of providing an explicit description of a set of generators of the maximal abelian extension $K^{a b}$ of a number field $K$ and an explicit description of the action of the Galois group $\operatorname{Gal}\left(K^{a b} / K\right)$ on them. This Galois group is the maximal abelian quotient of the absolute Galois group $\operatorname{Gal}(\bar{K} / K)$ of $K$, where $\bar{K}$ denotes an algebraic closure of $K$.

Remarkably, the only cases of number fields for which there is a complete answer to Hilbert's 12 th problem are the construction of the maximal abelian extension of $\mathbb{Q}$ using torsion points of $\mathbb{C}^{*}$ (KroneckerWeber) and the case of imaginary quadratic fields, where the construction relies on the theory of elliptic curves with complex multiplication (cf. e.g. the survey [33]).

If $\mathbb{A}_{K}$ denotes the adèles of a number field $K$ and $J_{K}=\mathrm{GL}_{1}\left(\mathbb{A}_{K}\right)$ is the group of idèles of $K$, we write $C_{K}$ for the group of idèle classes $C_{K}=J_{K} / K^{*}$ and $D_{K}$ for the connected component of the identity in $C_{K}$.

\subsection{Fabulous states for number fields.}

We discuss here briefly the relation of Problem 1.1 to Hilbert's 12 th problem, by concentrating on the arithmetic properties of the action of symmetries on the set $\mathcal{E}_{\infty}$ of extremal zero temperature KMS states. We abstract these properties in the notion of "fabulous states" discussed below.

Given a number field $K$, with a choice of an embedding $K \subset \mathbb{C}$, the "problem of fabulous states" consists of the following question.

Problem 2.3. Construct a $C^{*}$-dynamical system $\left(\mathcal{A}, \sigma_{t}\right)$, with an arithmetic subalgebra $\mathcal{A}_{K}$ of $\mathcal{A}$, with the following properties:

(1) The quotient group $G=C_{K} / D_{K}$ acts on $\mathcal{A}$ as symmetries compatible with $\sigma_{t}$ and preserving $\mathcal{A}_{K}$ 
(2) The states $\varphi \in \mathcal{E}_{\infty}$, evaluated on elements of the arithmetic subalgebra $\mathcal{A}_{K}$, satisfy:

- $\varphi(a) \in \bar{K}$, the algebraic closure of $K$ in $\mathbb{C}$;

- the elements of $\left\{\varphi(a): a \in \mathcal{A}_{K}, \varphi \in \mathcal{E}_{\infty}\right\}$ generate $K^{a b}$.

(3) The class field theory isomorphism

$$
\theta: C_{K} / D_{K} \stackrel{\simeq}{\longrightarrow} \operatorname{Gal}\left(K^{a b} / K\right)
$$

intertwines the actions,

$$
\alpha(\varphi(a))=\left(\varphi \circ \theta^{-1}(\alpha)\right)(a),
$$

for all $\alpha \in \operatorname{Gal}\left(K^{a b} / K\right)$, for all $\varphi \in \mathcal{E}_{\infty}$, and for all $a \in \mathcal{A}_{K}$.

(4) The algebra $\mathcal{A}_{K}$ has an explicit presentation by generators and relations.

It is important to make a general remark about Problem 2.3 versus the original Hilbert's 12 th problem. It may seem at first that the formulation of Problem 2.3 will not lead to any new information about the Hilbert 12 th problem. In fact, one can always consider here a "trivial system" where $\mathcal{A}=C\left(C_{K} / D_{K}\right)$ and $\sigma_{t}=i d$. All states are KMS states and the extremal ones are just valuations at points. Then one can always choose as the "rational subalgebra" the field $K^{a b}$ itself, embedded in the space of smooth functions on $C_{K} / D_{K}$ as $x \mapsto f_{x}(g)=\theta(g)(x)$, for $x \in K^{a b}$. Then this trivial system tautologically satisfies all the properties of Problem 2.3, except the last one. This last property, for this system, is then exactly as difficult as the original Hilbert 12th problem. This appears to show that the construction of "fabulous states" is just a reformulation of the original problem and need not simplify the task of obtaining explicit information about the generators of $K^{a b}$ and the Galois action.

The point is precisely that such "trivial example" is only a reformulation of the Hilbert 12th problem, while Problem 2.3 allows for nontrivial constructions of quantum statistical mechanical systems, where one can use essentially the fact of working with algebras instead of fields. The hope is that, for suitable systems, giving a presentation of the algebra $\mathcal{A}_{K}$ will prove to be an easier problem than giving generators of the field $K^{a b}$ and similarly for the action by symmetries on the algebra as opposed to the Galois action on the field.

Solutions to Problem 1.1 give nontrivial quantum statistical mechanical systems with the right Galois symmetries and arithmetic properties of zero temperature KMS states. (Notice that the trivial example above certainly will not satisfy the other properties sought for in Problem 1.1.) The hope is that, for such systems, the problem of giving a presentation of the algebra $\mathcal{A}_{K}$ may turn out to be easier than the original Hilbert 12th problem. We show in Proposition 3.3 in Section 3.3 below how, in the case of $K=\mathbb{Q}$, using the algebra of the BC system of [3], one can obtain a very simple description of $\mathbb{Q}^{a b}$ that uses only the explicit presentation of the algebra, without any reference to field extensions. This is possible precisely because the algebra is larger than the part that corresponds to the trivial system.

A broader type of question, in a similar spirit, can be formulated regarding the construction of quantum statistical mechanical systems with adèlic groups of symmetries and the arithmetic properties of its action on zero temperature extremal KMS states. The case of the $\mathrm{GL}_{2}$-system of [10] fits into this general program.

\subsection{Noncommutative pro-varieties.}

In the setting above, the $C^{*}$-dynamical system $\left(\mathcal{A}, \sigma_{t}\right)$ together with a $\mathbb{Q}$-structure compatible with the flow $\sigma_{t}$ (i.e. a rational subalgebra $\mathcal{A}_{\mathbb{Q}} \subset \mathcal{A}$ such that $\left.\sigma_{t}\left(\mathcal{A}_{\mathbb{Q}} \otimes \mathbb{C}\right)=\mathcal{A}_{\mathbb{Q}} \otimes \mathbb{C}\right)$ defines a non-commutative algebraic (pro-)variety $X$ over $\mathbb{Q}$. The ring $\mathcal{A}_{\mathbb{Q}}\left(\right.$ or $\left.\mathcal{A}_{\mathbb{Q}} \otimes \mathbb{C}\right)$, which need not be involutive, is the analog of the ring of algebraic functions on $X$ and the set of extremal $\mathrm{KMS}_{\infty}$-states is the analog of the set of points of $X$. As we will see for example in (3.3) and (3.31) below, the action of the subgroup of $\operatorname{Aut}\left(\mathcal{A}, \sigma_{t}\right)$ which takes $\mathcal{A}_{\mathbb{Q}} \otimes \mathbb{C}$ into itself is analogous to the action of the Galois group on the (algebraic) values of algebraic functions at points of $X$. 
The analogy illustrated above leads us to speak somewhat loosely of "classical points" of such a noncommutative algebraic pro-variety. We do not attempt to give a general definition of classical points, while we simply remark that, for the specific construction considered here, such a notion is provided by the zero temperature extremal states.

\section{Q-Lattices and noncommutative Shimura Varieties}

In this section we recall the main properties of the $\mathrm{BC}$ and the $\mathrm{GL}_{2}$ system, which will be useful for our main result.

Both cases can be formulated starting with the same geometric notion, that of commensurability classes of $\mathbb{Q}$-lattices, in dimension one and two, respectively.

Definition 3.1. $A \mathbb{Q}$-lattice in $\mathbb{R}^{n}$ is a pair $(\Lambda, \phi)$, with $\Lambda$ a lattice in $\mathbb{R}^{n}$, and

$$
\phi: \mathbb{Q}^{n} / \mathbb{Z}^{n} \longrightarrow \mathbb{Q} \Lambda / \Lambda
$$

a homomorphism of abelian groups. $A \mathbb{Q}$-lattice is invertible if the map (3.1) is an isomorphism. Two $\mathbb{Q}$-lattices $\left(\Lambda_{1}, \phi_{1}\right)$ and $\left(\Lambda_{2}, \phi_{2}\right)$ are commensurable if the lattices are commensurable $\left(\right.$ i.e. $\left.\mathbb{Q} \Lambda_{1}=\mathbb{Q} \Lambda_{2}\right)$ and the maps agree modulo the sum of the lattices,

$$
\phi_{1} \equiv \phi_{2} \quad \bmod \Lambda_{1}+\Lambda_{2}
$$

It is essential here that one does not require the homomorphism $\phi$ to be invertible in general.

The set of $\mathbb{Q}$-lattices modulo the equivalence relation of commensurability is best described with the tools of noncommutative geometry, as explained in [10].

We will be concerned here only with the case of $n=1$ or $n=2$ and we will consider also the set of commensurability classes of $\mathbb{Q}$-lattices up to scaling, where the scaling action is given by the group $S=\mathbb{R}_{+}^{*}$ in the 1-dimensional case and $S=\mathbb{C}^{*}$ in the 2-dimensional case.

In these cases, one can first consider the groupoid $\mathcal{R}$ of the equivalence relation of commensurability on the set of $\mathbb{Q}$-lattices (not up to scaling). This is a locally compact étale groupoid. When considering the quotient by the scaling action, the algebra of coordinates associated to the quotient $\mathcal{R} / S$ is obtained by restricting the convolution product of the algebra of $\mathcal{R}$ to weight zero functions with $S$-compact support. The algebra obtained this way, which is unital in the 1-dimensional case, but not in the 2-dimensional case, has a natural time evolution given by the ratio of the covolumes of a pair of commensurable lattices. Every unit $y \in \mathcal{R}^{(0)}$ of $\mathcal{R}$ defines a representation $\pi_{y}$ by left convolution of the algebra of $\mathcal{R}$ on the Hilbert space $\mathcal{H}_{y}=\ell^{2}\left(\mathcal{R}_{y}\right)$, where $\mathcal{R}_{y}$ is the set of elements with source $y$. This construction passes to the quotient by the scaling action of $S$. Representations corresponding to points that acquire a nontrivial automorphism group will no longer be irreducible. If the unit $y \in \mathcal{R}^{(0)}$ corresponds to an invertible $\mathbb{Q}$-lattice, then $\pi_{y}$ is a positive energy representation.

In both the 1-dimensional and the 2-dimensional case, the set of extremal KMS states at low temperature is given by a classical adèlic quotient, namely, by the Shimura varieties for $\mathrm{GL}_{1}$ and $\mathrm{GL}_{2}$, respectively, hence we argue here that the noncommutative space describing commensurability classes of $\mathbb{Q}$-lattices up to scale can be thought of as a noncommutative Shimura variety, whose set of classical points is the corresponding classical Shimura variety.

In both cases, a crucial step for the arithmetic properties of the action of symmetries on extremal KMS states at zero temperature is the choice of an arithmetic subalgebra of the system, on which the extremal $\mathrm{KMS}_{\infty}$ states are evaluated. Such choice gives the underlying noncommutative space a more rigid structure, of "noncommutative arithmetic variety". 


\subsection{Tower Power.}

If $V$ is an algebraic variety - or a scheme or a stack - over a field $k$, a "tower" $\mathcal{T}$ over $V$ is a family $V_{i}$ $(i \in \mathcal{I})$ of finite (possibly branched) covers of $V$ such that for any $i, j \in \mathcal{I}$, there is a $l \in \mathcal{I}$ with $V_{l}$ a cover of $V_{i}$ and $V_{j}$. Thus, $\mathcal{I}$ is a partially ordered set. This gives a corresponding compatible system of covering maps $V_{i} \rightarrow V$. In case of a tower over a pointed variety $(V, v)$, one fixes a point $v_{i}$ over $v$ in each $V_{i}$. Even though $V_{i}$ may not be irreducible, we shall allow ourselves to loosely refer to $V_{i}$ as a variety. It is convenient to view a "tower" $\mathcal{T}$ as a category $\mathcal{C}$ with objects $\left(V_{i} \rightarrow V\right)$ and morphisms $\operatorname{Hom}\left(V_{i}, V_{j}\right)$ being maps of covers of $V$. One has the group $\operatorname{Aut}_{\mathcal{T}}\left(V_{i}\right)$ of invertible self-maps of $V_{i}$ over $V$ (the group of deck transformations); the deck transformations are not required to preserve the points $v_{i}$. There is a (profinite) group of symmetries associated to a tower, namely

$$
\mathcal{G}:=\lim _{i} \operatorname{Aut}_{\mathcal{T}}\left(V_{i}\right) .
$$

The simplest example of a tower is the "fundamental group" tower associated with a (smooth connected) complex algebraic variety $(V, v)$ and its universal covering $(\tilde{V}, \tilde{v})$. Let $\mathcal{C}$ be the category of all finite étale (unbranched) intermediate covers $\tilde{V} \rightarrow W \rightarrow V$ of $V$. In this case, the symmetry group $\mathcal{G}$ of (3.2) is the algebraic fundamental group of $V$, which is also the profinite completion of the (topological) fundamental group $\pi_{1}(V, v)$. (For the theory of étale covers and fundamental groups, we refer the interested reader to SGA1.) Simple variants of this example include allowing controlled ramification. Other examples of towers are those defined by iteration of self maps of algebraic varieties. For us, the most important examples of "towers" will be the cyclotomic tower and the modular tower ${ }^{1}$. Another very interesting case of towers is that of more general Shimura varieties. These, however, will not be treated in this paper. (For a systematic treatment of quantum statistical mechanical systems associated to general Shimura varieties see [13] and upcoming work by the same authors.)

\subsection{The cyclotomic tower and the BC system.}

In the case of $\mathbb{Q}$, an explicit description of $\mathbb{Q}^{a b}$ is provided by the Kronecker-Weber theorem. This shows that the field $\mathbb{Q}^{a b}$ is equal to $\mathbb{Q}^{c y c}$, the field obtained by attaching all roots of unity to $\mathbb{Q}$. Namely, $\mathbb{Q}^{a b}$ is obtained by attaching the values of the exponential function $\exp (2 \pi i z)$ at the torsion points of the circle group $\mathbb{R} / \mathbb{Z}$. Using the isomorphism of abelian groups $\overline{\mathbb{Q}}_{\text {tors }}^{*} \cong \mathbb{Q} / \mathbb{Z}$ and the identification $\operatorname{Aut}(\mathbb{Q} / \mathbb{Z})=\mathrm{GL}_{1}(\hat{\mathbb{Z}})=\hat{\mathbb{Z}}^{*}$, the restriction to $\overline{\mathbb{Q}}_{\text {tors }}^{*}$ of the natural action of $\operatorname{Gal}(\overline{\mathbb{Q}} / \mathbb{Q})$ on $\overline{\mathbb{Q}}^{*}$ factors as

$$
\operatorname{Gal}(\overline{\mathbb{Q}} / \mathbb{Q}) \rightarrow \operatorname{Gal}(\overline{\mathbb{Q}} / \mathbb{Q})^{a b}=\operatorname{Gal}\left(\mathbb{Q}^{a b} / \mathbb{Q}\right) \stackrel{\sim}{\longrightarrow} \hat{\mathbb{Z}}^{*}
$$

Geometrically, the above setting can be understood in terms of the cyclotomic tower. This has base Spec $\mathbb{Z}=V_{1}$. The family is Spec $\mathbb{Z}\left[\zeta_{n}\right]=V_{n}$ where $\zeta_{n}$ is a primitive $n$-th root of unity $\left(n \in \mathbb{N}^{*}\right)$. The set Hom $\left(V_{m} \rightarrow V_{n}\right)$, non-trivial for $n \mid m$, corresponds to the map $\mathbb{Z}\left[\zeta_{n}\right] \hookrightarrow \mathbb{Z}\left[\zeta_{m}\right]$ of rings. The group $\operatorname{Aut}\left(V_{n}\right)=\mathrm{GL}_{1}(\mathbb{Z} / n \mathbb{Z})$ is the Galois group $\operatorname{Gal}\left(\mathbb{Q}\left(\zeta_{n}\right) / \mathbb{Q}\right)$. The group of symmetries $(3.2)$ of the tower is then

$$
\mathcal{G}={\underset{\leftarrow}{n}}_{\lim _{1}} \mathrm{GL}_{1}(\mathbb{Z} / n \mathbb{Z})=\mathrm{GL}_{1}(\hat{\mathbb{Z}})
$$

which is isomorphic to the Galois group $\operatorname{Gal}\left(\mathbb{Q}^{a b} / \mathbb{Q}\right)$ of the maximal abelian extension of $\mathbb{Q}$.

The classical object that we consider, associated to the cyclotomic tower, is the Shimura variety given by the adèlic quotient

$$
\operatorname{Sh}\left(\mathrm{GL}_{1},\{ \pm 1\}\right)=\mathrm{GL}_{1}(\mathbb{Q}) \backslash\left(\mathrm{GL}_{1}\left(\mathbb{A}_{f}\right) \times\{ \pm 1\}\right)=\mathbb{A}_{f}^{*} / \mathbb{Q}_{+}^{*} .
$$

Now we consider the space of 1-dimensional $\mathbb{Q}$-lattices up to scaling modulo commensurability. This can be described as follows $([10])$.

\footnotetext{
${ }^{1}$ In this paper, we reserve the terminology "modular tower" for the tower of modular curves. It would be interesting to investigate in a similar perspective the more general theory of modular towers in the sense of M.Fried.
} 
In one dimension, every $\mathbb{Q}$-lattice is of the form

$$
(\Lambda, \phi)=(\lambda \mathbb{Z}, \lambda \rho)
$$

for some $\lambda>0$ and some $\rho \in \operatorname{Hom}(\mathbb{Q} / \mathbb{Z}, \mathbb{Q} / \mathbb{Z})$. Since we can identify $\operatorname{Hom}(\mathbb{Q} / \mathbb{Z}, \mathbb{Q} / \mathbb{Z})$ endowed with the topology of pointwise convergence with

$$
\operatorname{Hom}(\mathbb{Q} / \mathbb{Z}, \mathbb{Q} / \mathbb{Z})=\overleftarrow{n}_{n}^{\lim } \mathbb{Z} / n \mathbb{Z}=\hat{\mathbb{Z}},
$$

we obtain that the algebra $C(\hat{\mathbb{Z}})$ is the algebra of coordinates of the space of 1 -dimensional $\mathbb{Q}$-lattices up to scaling.

In fact, using the identification

$$
\mathbb{Q} / \mathbb{Z} \cong \mathbb{A}_{f} / \hat{\mathbb{Z}}
$$

one gets a natural character $e$ of $\mathbb{A}_{f} / \hat{\mathbb{Z}}$ such that

$$
e(r)=e^{2 \pi i r} \quad \forall r \in \mathbb{Q} / \mathbb{Z}
$$

and a pairing of $\mathbb{Q} / \mathbb{Z}$ with $\hat{\mathbb{Z}}$ such that

$$
\langle r, x\rangle=e(r x) \quad \forall r \in \mathbb{Q} / \mathbb{Z}, x \in \hat{\mathbb{Z}}
$$

Thus, we identify the group $\hat{\mathbb{Z}}$ with the Pontrjagin dual of $\mathbb{Q} / \mathbb{Z}$ and we obtain a corresponding identification

$$
C^{*}(\mathbb{Q} / \mathbb{Z}) \cong C(\hat{\mathbb{Z}})
$$

The group of deck transformations $\mathcal{G}=\hat{\mathbb{Z}}^{*}$ of the cyclotomic tower acts by automorphisms on the algebra of coordinates $C(\hat{\mathbb{Z}})$ in the obvious way. In addition to this action, there is a semigroup action of $\mathbb{N}^{\times}=\mathbb{Z}_{>0}$ implementing the commensurability relation. This is given by endomorphisms that move vertically across the levels of the cyclotomic tower. They are given by

$$
\alpha_{n}(f)(\rho)= \begin{cases}f\left(n^{-1} \rho\right), & \rho \in n \hat{\mathbb{Z}} \\ 0 & \text { otherwise. }\end{cases}
$$

Namely, $\alpha_{n}$ is the isomorphism of $C(\hat{\mathbb{Z}})$ with the reduced algebra $C(\hat{\mathbb{Z}})_{\pi_{n}}$ by the projection $\pi_{n}$ given by the characteristic function of $n \hat{\mathbb{Z}} \subset \mathbb{Z}$. Notice that the action (3.8) cannot be restricted to the set of invertible $\mathbb{Q}$-lattices, since the range of $\pi_{n}$ is disjoint from them.

The algebra of coordinates $\mathcal{A}_{1}$ on the noncommutative space of equivalence classes of 1-dimensional $\mathbb{Q}$-lattices modulo scaling, with respect to the equivalence relation of commensurability, is given then by the semigroup crossed product

$$
\mathcal{A}=C(\hat{\mathbb{Z}}) \rtimes_{\alpha} \mathbb{N}^{\times}
$$

Equivalently, we are considering the convolution algebra of the groupoid $\mathcal{R}_{1} / \mathbb{R}_{+}^{*}$ given by the quotient by scaling of the groupoid of the equivalence relation of commensurability on 1-dimensional $\mathbb{Q}$-lattices, namely, $\mathcal{R}_{1} / \mathbb{R}_{+}^{*}$ has as algebra of coordinates the functions $f(r, \rho)$, for $\rho \in \hat{\mathbb{Z}}$ and $r \in \mathbb{Q}^{*}$ such that $r \rho \in \hat{\mathbb{Z}}$, where $r \rho$ is the product in $\mathbb{A}_{f}$. The product in the algebra is given by the associative convolution product

$$
f_{1} * f_{2}(r, \rho)=\sum_{s: s \rho \in \hat{\mathbb{Z}}} f_{1}\left(r s^{-1}, s \rho\right) f_{2}(s, \rho),
$$

and the adjoint is given by $f^{*}(r, \rho)=\overline{f\left(r^{-1}, r \rho\right)}$.

This is the $C^{*}$-algebra of the Bost-Connes (BC) system [3]. It has a natural time evolution $\sigma_{t}$ determined by the ratio of the covolumes of two commensurable $\mathbb{Q}$-lattices,

$$
\sigma_{t}(f)(r, \rho)=r^{i t} f(r, \rho) .
$$

The algebra $\mathcal{A}_{1}$ was originally defined as a Hecke algebra for the almost normal pair of solvable groups $P_{\mathbb{Z}}^{+} \subset P_{\mathbb{Q}}^{+}$, where $P$ is the algebraic $a x+b$ group and $P^{+}$is the restriction to $a>0(c f .[3])$. 
As a set, the space of commensurability classes (cf. Definition 3.1) of 1-dimensional $\mathbb{Q}$-lattices up to scaling can also be described by the quotient

$$
\mathrm{GL}_{1}(\mathbb{Q}) \backslash \mathbb{A}^{*} / \mathbb{R}_{+}^{*}=\mathrm{GL}_{1}(\mathbb{Q}) \backslash\left(\mathbb{A}_{f} \times\{ \pm 1\}\right)=\mathbb{A}_{f} / \mathbb{Q}_{+}^{*},
$$

where $\mathbb{A}^{*}:=\mathbb{A}_{f} \times \mathbb{R}^{*}$ is the set of adèles with nonzero archimedean component. Rather than considering this quotient set theoretically, we regard it as a noncommutative space, so as to be able to extend to it the ordinary tools of geometry that can be applied to the "good" quotient (3.4).

The noncommutative algebra of coordinates of (3.12) is the crossed product

$$
C_{0}\left(\mathbb{A}_{f}\right) \rtimes \mathbb{Q}_{+}^{*} .
$$

This is Morita equivalent to the algebra (3.9). In fact, (3.9) is obtained as a full corner of (3.13),

$$
C(\hat{\mathbb{Z}}) \rtimes \mathbb{N}^{\times}=\left(C_{0}\left(\mathbb{A}_{f}\right) \rtimes \mathbb{Q}_{+}^{*}\right)_{\pi},
$$

by compression with the projection $\pi$ given by the characteristic function of $\hat{\mathbb{Z}} \subset \mathbb{A}_{f}(c f$. [19]).

The quotient (3.12) with its noncommutative algebra of coordinates (3.13) can then be thought of as the noncommutative Shimura variety

$$
S h^{(n c)}\left(\mathrm{GL}_{1},\{ \pm 1\}\right):=\mathrm{GL}_{1}(\mathbb{Q}) \backslash\left(\mathbb{A}_{f} \times\{ \pm 1\}\right)=\mathrm{GL}_{1}(\mathbb{Q}) \backslash \mathbb{A}^{*} / \mathbb{R}_{+}^{*},
$$

whose set of classical points is the well behaved quotient (3.4).

The noncommutative space of adèle classes, used in [8] in order to obtain the spectral realization of the zeros of the Riemann zeta function, is closely related to the noncommutative Shimura variety (3.14). First, one replaces $\mathbb{A}^{\cdot}$ with $\mathbb{A}$, as in [8]. This means adding the trivial lattice (the point $\lambda=0$ in the $\mathbb{R}$-component of $\mathbb{A}$ ), with a possibly nontrivial $\mathbb{Q}$-structure (given by some $\rho \neq 0$ in $\mathbb{A}_{f}$ ). One obtains this way the space

$$
\mathrm{GL}_{1}(\mathbb{Q}) \backslash \mathbb{A} / \mathbb{R}_{+}^{*} .
$$

One then obtains the space of adèle classes of [8] as the dual to (3.15) under the duality given by taking the crossed product by the time evolution (i.e. in the duality between type II and type III factors of $[6]$ ). The space obtained this way is a principal $\mathbb{R}_{+}^{*}$-bundle over the noncommutative space

$$
\mathrm{GL}_{1}(\mathbb{Q}) \backslash \mathbb{A} / \mathbb{R}_{+}^{*} \text {. }
$$

\subsection{Arithmetic structure of the BC system.}

The results of [3] show that the Galois theory of the cyclotomic field $\mathbb{Q}^{c y c l}$ appears naturally in the $\mathrm{BC}$ system when considering the action of the group of symmetries of the system on the extremal KMS states at zero temperature.

In the case of 1-dimensional $\mathbb{Q}$-lattices up to scaling, the algebra of coordinates $C(\hat{\mathbb{Z}})$ can be regarded as the algebra of homogeneous functions of weight zero on the space of 1 -dimensional $\mathbb{Q}$-lattices. As such, there is a natural choice of an arithmetic subalgebra.

This is obtained in [10] by considering functions on the space of 1-dimensional $\mathbb{Q}$-lattices of the form

$$
\epsilon_{1, a}(\Lambda, \phi)=\sum_{y \in \Lambda+\phi(a)} y^{-1}
$$

for any $a \in \mathbb{Q} / \mathbb{Z}$. This is well defined, for $\phi(a) \neq 0$, using the summation $\lim _{N \rightarrow \infty} \sum_{-N}^{N}$. One can then form the weight zero functions

$$
e_{1, a}:=c \epsilon_{1, a},
$$

where $c(\Lambda)$ is proportional to the covolume $|\Lambda|$ and normalized so that $(2 \pi \sqrt{-1}) c(\mathbb{Z})=1$. The rational subalgebra $\mathcal{A}_{1, \mathbb{Q}}$ of $(3.9)$ is the $\mathbb{Q}$-algebra generated by the functions $e_{1, a}(r, \rho):=e_{1, a}(\rho)$ and by the functions $\mu_{n}(r, \rho)=1$ for $r=n$ and zero otherwise. The latter implement the semigroup action of $\mathbb{N}^{\times}$in $(3.9)$. 
As proved in [10], the algebra $\mathcal{A}_{1, \mathbb{Q}}$ is the same as the rational subalgebra considered in [3], generated over $\mathbb{Q}$ by the $\mu_{n}$ and the exponential functions

$$
e(r)(\rho):=\exp (2 \pi i \rho(r)), \quad \text { for } \rho \in \operatorname{Hom}(\mathbb{Q} / \mathbb{Z}, \mathbb{Q} / \mathbb{Z}), \quad \text { and } r \in \mathbb{Q} / \mathbb{Z},
$$

with relations $e(r+s)=e(r) e(s), e(0)=1, e(r)^{*}=e(-r), \mu_{n}^{*} \mu_{n}=1, \mu_{k} \mu_{n}=\mu_{k n}$, and

$$
\mu_{n} e(r) \mu_{n}^{*}=\frac{1}{n} \sum_{n s=r} e(s) .
$$

The $C^{*}$-completion of $\mathcal{A}_{1, \mathbb{Q}} \otimes \mathbb{C}$ gives (3.9).

The algebra (3.9) has irreducible representations on the Hilbert space $\mathcal{H}=\ell^{2}\left(\mathbb{N}^{\times}\right)$, parameterized by elements $\alpha \in \hat{\mathbb{Z}}^{*}=\mathrm{GL}_{1}(\hat{\mathbb{Z}})$. Any such element defines an embedding $\alpha: \mathbb{Q}^{\text {cycl }} \hookrightarrow \mathbb{C}$ and the corresponding representation is of the form

$$
\pi_{\alpha}(e(r)) \epsilon_{k}=\alpha\left(\zeta_{r}^{k}\right) \epsilon_{k} \quad \pi_{\alpha}\left(\mu_{n}\right) \epsilon_{k}=\epsilon_{n k} .
$$

The Hamiltonian implementing the time evolution $\sigma_{t}$ on $\mathcal{H}$ is of the form $H \epsilon_{k}=\log k \epsilon_{k}$ and the partition function of the $\mathrm{BC}$ system is then the Riemann zeta function

$$
Z(\beta)=\operatorname{Tr}\left(e^{-\beta H}\right)=\sum_{k=1}^{\infty} k^{-\beta}=\zeta(\beta) .
$$

The set $\mathcal{E}_{\beta}$ of extremal KMS-states of the BC system enjoys the following properties ( $c f$. [3]):

Theorem 3.2. (Bost-Connes [3]) For the system $\left(\mathcal{A}_{1}, \sigma_{t}\right)$ described above, the structure of KMS states is the following.

- $\mathcal{E}_{\beta}$ is a singleton for all $0<\beta \leq 1$. This unique KMS state takes values

$$
\varphi_{\beta}(e(m / n))=f_{-\beta+1}(n) / f_{1}(n),
$$

where

$$
f_{k}(n)=\sum_{d \mid n} \mu(d)(n / d)^{k},
$$

with $\mu$ the Möbius function, and $f_{1}$ is the Euler totient function.

- For $1<\beta \leq \infty$, elements of $\mathcal{E}_{\beta}$ are indexed by the classes of invertible $\mathbb{Q}$-lattices $\rho \in \hat{\mathbb{Z}}^{*}=$ $\mathrm{GL}_{1}(\hat{\mathbb{Z}})$, hence by the classical points (3.4) of the noncommutative Shimura variety (3.14),

$$
\mathcal{E}_{\beta} \cong \mathrm{GL}_{1}(\mathbb{Q}) \backslash \mathrm{GL}_{1}(\mathbb{A}) / \mathbb{R}_{+}^{*} \cong C_{\mathbb{Q}} / D_{\mathbb{Q}} \cong \mathbb{I}_{\mathbb{Q}} / \mathbb{Q}_{+}^{*}
$$

with $\mathbb{I}_{\mathbb{Q}}$ as in (1.1). In this range of temperatures, the values of states $\varphi_{\beta, \rho} \in \mathcal{E}_{\beta}$ on the elements $e(r) \in \mathcal{A}_{1, \mathbb{Q}}$ is given, for $1<\beta<\infty$ by polylogarithms evaluated at roots of unity, normalized by the Riemann zeta function,

$$
\varphi_{\beta, \rho}(e(r))=\frac{1}{\zeta(\beta)} \sum_{n=1}^{\infty} n^{-\beta} \rho\left(\zeta_{r}^{k}\right) .
$$

- The group $\mathrm{GL}_{1}(\hat{\mathbb{Z}})$ acts by automorphisms of the system. The induced action of $G L_{1}(\hat{\mathbb{Z}})$ on the set of extreme KMS states below critical temperature is free and transitive.

- The extreme KMS states at $(\beta=\infty)$ are fabulous states for the field $K=\mathbb{Q}$, namely $\varphi\left(\mathcal{A}_{1, \mathbb{Q}}\right) \subset \mathbb{Q}^{c y c l}$ and the class field theory isomorphism $\theta: \operatorname{Gal}\left(\mathbb{Q}^{\text {cycl }} / \mathbb{Q}\right) \stackrel{\cong}{\cong} \hat{\mathbb{Z}}^{*}$ intertwines the Galois action on values with the action of $\hat{\mathbb{Z}}^{*}$ by symmetries,

$$
\gamma \varphi(x)=\varphi(\theta(\gamma) x)
$$

for all $\varphi \in \mathcal{E}_{\infty}$, for all $\gamma \in \operatorname{Gal}\left(\mathbb{Q}^{\text {cycl }} / \mathbb{Q}\right)$ and for all $x \in \mathcal{A}_{1, \mathbb{Q}}$. 
This shows that the BC system is a solution to Problem 1.1 for $K=\mathbb{Q}$.

We return now to the discussion of Section 2.3 about the comparison between the problem of fabulous states (Problem 2.3) and explicit class field theory. In the case of the BC system, the "trivial system" mentioned in Section 2.3 corresponds to considering the abelian part of the algebra restricted to invertible $\mathbb{Q}$-lattices. On this the restriction of the time evolution is trivial. The simple transitivity of the action of the Galois group on $S$ shows trivially that this restriction $\mathcal{B}$ is isomorphic to $\mathbb{Q}^{a b}$, viewed as a subalgebra of $C(S)$.

It is important to understand, already in the case of the BC system, how the "rational subalgebra" $\mathcal{A}_{1, \mathbb{Q}}$ is simpler than the restriction $\mathcal{B}$ of its abelian part to the space $S \cong \mathbb{A}_{f}^{*} / \mathbb{Q}_{+}^{*}$ of invertible $\mathbb{Q}$ lattices. This depends essentially on the fact of considering all $\mathbb{Q}$-lattices, not just the invertible ones.

First one can see easily that the "trivial system" $\left(\mathcal{B}, \sigma_{t}\right)$ obtained this way is no longer a solution to Problem 1.1, as it does not fulfill any of the deeper properties regarding phase transitions and the partition function. However, the main point we wish to discuss here is the fact that, passing from $\mathcal{B} \cong \mathbb{Q}^{a b}$ to the algebra $\mathcal{A}_{1, \mathbb{Q}}$ gives us a simpler presentation.

The presentation of $\mathbb{Q}^{a b}$ is given by the cyclotomic theory, while our rational algebra is simply the group ring of $\mathbb{Q} / \mathbb{Z}$ with coefficients in $\mathbb{Q}$. We can use this to obtain a presentation of $\mathbb{Q}^{a b}$ which does not use any direct reference to field extensions in the following way.

Let $H=\mathbb{Q} / \mathbb{Z}$ be the additive group given by the quotient of $\mathbb{Q}$ by $\mathbb{Z} \subset \mathbb{Q}$. Consider the inclusion of algebras

$$
\mathbb{Q}[H] \subset C^{*}(H)
$$

and let $J \subset C^{*}(H)$ be the ideal generated by the idempotents

$$
\pi_{m}=\frac{1}{m} \sum_{r \in H, m r=0} u(r),
$$

where $m>1$ and the $u(r)$ form the canonical basis of $\mathbb{Q}[H]$.

Proposition 3.3. With $H$ and $J$ as above, the following holds.

(1) The quotient

$$
\mathbb{Q}[H] /(\mathbb{Q}[H] \cap J)
$$

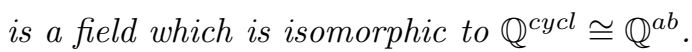

(2) $\mathbb{Q}[H] \cap J$ is equal to the ideal generated by the $\pi_{m}$ in $\mathbb{Q}[H]$.

Proof. 1) We use the identification of the group $\hat{\mathbb{Z}}$ with the Pontrjagin dual of $H$ and the isomorphism of algebras (3.7).

Under this identification, the ideal $J$ corresponds to the ideal of functions that vanish on the closed subset

$$
\hat{\mathbb{Z}}^{*} \subset \hat{\mathbb{Z}} \text {. }
$$

The quotient map $C^{*}(H) \rightarrow C^{*}(H) / J$ is just the restriction map

$$
\left.f \in C(\hat{\mathbb{Z}}) \mapsto f\right|_{\hat{\mathbb{Z}}^{*}} .
$$

In fact, first notice that the restriction of $\pi_{m}$ to $\hat{\mathbb{Z}}^{*}$ vanishes since the sum of all roots of unit of order $m$ is zero. This shows that $J$ is contained in the kernel of the restriction map (3.27). Moreover, a character of the $C^{*}$-algebra $C^{*}(H) / J$ is given by a point $a \in \hat{\mathbb{Z}}$ on which all elements of $J$ vanish. However, if $a \notin \hat{\mathbb{Z}}^{*}$, then there exists $m>1$ such that $a \in m \times \hat{\mathbb{Z}}$ and one gets $\pi_{m}(a)=1 \neq 0$.

The multiplicative group $G=\hat{\mathbb{Z}}^{*}$ acts by multiplication on both $\mathbb{A}_{f} / \hat{\mathbb{Z}} \cong H$ and on $\hat{\mathbb{Z}}$ and these actions are compatible with the pairing

$$
\langle g r, x\rangle=\langle r, g x\rangle
$$


and therefore with the isomorphism (3.7), and with the actions of $G$ by automorphisms

$$
G \subset \text { Aut } C^{*}(H), \quad G \subset \text { Aut } C(\hat{\mathbb{Z}}) .
$$

The group $G$ is also identified with the Galois group of the cyclotomic field, i.e. the subfield $\mathbb{Q}^{c y c} \subset \mathbb{C}$ generated by all the roots of unity of all orders. For any element $v \in \hat{\mathbb{Z}}^{*} \subset \hat{\mathbb{Z}}$, let $\varphi_{v}$ denote the evaluation map $\varphi_{v}: C(\hat{\mathbb{Z}}) \rightarrow \mathbb{C}$, with $\varphi_{v}(f)=f(v)$. These maps fulfill the condition

$$
g\left(\varphi_{v}(f)\right)=\varphi_{v}(g(f))=\varphi_{g v}(f) .
$$

for all $g \in G$ and for all $f \in \mathbb{Q}[H]$, where we use the inclusion (3.24) and the identification (3.7).

Thus, the restriction of $f \in \mathbb{Q}[H]$ to $\hat{\mathbb{Z}}^{*} \subset \hat{\mathbb{Z}}$ is determined by the single field element $\varphi_{1}(f) \in \mathbb{Q}^{c y c}$. The restriction map (3.27) then induces an isomorphism

$$
\mathbb{Q}[H] /(\mathbb{Q}[H] \cap J) \stackrel{\cong}{\longrightarrow} \mathbb{Q}^{c y c} .
$$

2) Let $J_{0}$ be the ideal generated by the $\pi_{m}$ in $\mathbb{Q}[H]$. It is enough to show that, for $\Phi_{n}$ the $n$-th cyclotomic polynomial ( $c f$. [26]), one has $\Phi_{n}(u(1 / n)) \in J_{0}$. When $n$ is prime one has $\Phi_{n}(u(1 / n))=$ $n \pi_{n}$. In general, if we write

$$
\sigma_{k}(x)=\sum_{0}^{k-1} x^{j},
$$

then we obtain that $\Phi_{n}$ is the g.c.d. of the polynomials $\sigma_{m}\left(x^{d}\right)$, where $d$ divides $n$ and $m=n / d$. For $x=u(1 / n)$, one has $\sigma_{m}\left(x^{d}\right)=m \pi_{m} \in J_{0}$, for any divisor $d \mid n$. Thus, we obtain $\Phi_{n}(u(1 / n)) \in J_{0}$ as required.

Recall that $\pi_{m}=\mu_{m} \mu_{m}^{*}$ and $\mu_{m}^{*} \mu_{m}=1$. Thus, the $\mathrm{KMS}_{\infty}$ states of the BC system vanish identically on the $\pi_{m}$. The $\mathrm{KMS}_{\beta}$ condition for $1<\beta<\infty$ shows that $\mathrm{KMS}_{\beta}$ states take value $m^{-\beta}$ on $\pi_{m}$. It follows that the restriction of $\mathrm{KMS}_{\infty}$ states to the abelian part vanish identically on $J$.

In our setup we proved in $[10]$ that the rational subalgebra $\mathcal{A}_{1, \mathbb{Q}}$ of the BC system is generated by the natural simple functions of $\mathbb{Q}$-lattices given by summation and is actually the same as $\mathbb{Q}[H] \subset C^{*}(H)$. (This follows using the isomorphism of $C^{*}(H)$ with continuous functions of weight zero on $\mathbb{Q}$-lattices.) Thus, one can reasonably expect that similar simplifications will occur in the case of imaginary quadratic fields.

In [10] we described a set of non-trivial relations for the algebra $\mathcal{A}_{2, \mathbb{Q}}$ of the $\mathrm{GL}_{2}$-system (which will be recalled in Definition 3.8 below). One can consider the specialization of these relations to the value of the $j$-invariant of an elliptic curve with complex multiplication will provide relations for the CM system (described in Section 4 below). We expect that this will provide the for an explicit presentation of the rational subalgebra $\mathcal{A}_{K, \mathbb{Q}}$ of the CM system as an algebra over the Hilbert class field $K(j)$.

\subsection{The modular tower and the $\mathrm{GL}_{2}$-system.}

Modular curves arise as moduli spaces of elliptic curves endowed with additional level structure. Every congruence subgroup $\Gamma^{\prime}$ of $\Gamma=\mathrm{SL}_{2}(\mathbb{Z})$ defines a modular curve $Y_{\Gamma^{\prime}}$; we denote by $X_{\Gamma^{\prime}}$ the smooth compactification of the affine curve $Y_{\Gamma^{\prime}}$ obtained by adding cusp points. Especially important among these are the modular curves $Y(n)$ and $X(n)$ corresponding to the principal congruence subgroups $\Gamma(n)$ for $n \in \mathbb{N}^{*}$. Any $X_{\Gamma^{\prime}}$ is dominated by an $X(n)$. We refer to $[17,32]$ for more details. We have the following descriptions of the modular tower.

Compact version: The base is $V=\mathbb{P}^{1}$ over $\mathbb{Q}$. The family is given by the modular curves $X(n)$, considered over the cyclotomic field $\mathbb{Q}\left(\zeta_{n}\right)[25]$. We note that $\mathrm{GL}_{2}(\mathbb{Z} / n \mathbb{Z}) / \pm 1$ is the group of automorphisms of the projection $V_{n}=X(n) \rightarrow X(1)=V_{1}=V$. Thus, we have

$$
\mathcal{G}=\mathrm{GL}_{2}(\hat{\mathbb{Z}}) /\{ \pm 1\}=\underset{\leftarrow}{\lim } \mathrm{GL}_{2}(\mathbb{Z} / n \mathbb{Z}) /\{ \pm 1\}
$$


Non-compact version: The open modular curves $Y(n)$ form a tower with base the $j$-line Spec $\mathbb{Q}[j]=$ $\mathbb{A}^{1}=V_{1}-\{\infty\}$. The ring of modular functions is the union of the rings of functions of the $Y(n)$, with coefficients in $\mathbb{Q}\left(\zeta_{n}\right)[17]$.

This shows how the modular tower is a natural geometric way of passing from $\operatorname{GL}_{1}(\hat{\mathbb{Z}})$ to $\mathrm{GL}_{2}(\hat{\mathbb{Z}})$. The formulation that is most convenient in our setting is the one given in terms of Shimura varieties. In fact, rather than the modular tower defined by the projective limit

$$
Y=\lim _{n} Y(n)
$$

of the modular curves $Y(n)$, it is better for our purposes to consider the Shimura variety

$$
S h\left(\mathbb{H}^{ \pm}, \mathrm{GL}_{2}\right)=\mathrm{GL}_{2}(\mathbb{Q}) \backslash\left(\mathrm{GL}_{2}\left(\mathbb{A}_{f}\right) \times \mathbb{H}^{ \pm}\right)=\mathrm{GL}_{2}(\mathbb{Q}) \backslash \mathrm{GL}_{2}(\mathbb{A}) / \mathbb{C}^{*},
$$

of which (3.32) is a connected component. Here $\mathbb{H}^{ \pm}$denotes the upper and lower half planes.

It is well known that, for arithmetic purposes, it is always better to work with nonconnected rather than with connected Shimura varieties ( $c f$. e.g. [25]). The simple reason why it is necessary to pass to the nonconnected case is the following. The varieties in the tower are arithmetic varieties defined over number fields. However, the number field typically changes along the levels of the tower $(Y(n)$ is defined over the cyclotomic field $\mathbb{Q}\left(\zeta_{n}\right)$ ). Passing to nonconnected Shimura varieties allows precisely for the definition of a canonical model where the whole tower is defined over the same number field.

This distinction is important to our viewpoint, since we want to work with noncommutative spaces endowed with an arithmetic structure, specified by the choice of an arithmetic subalgebra.

Every 2-dimensional $\mathbb{Q}$-lattice can be described by data

$$
(\Lambda, \phi)=(\lambda(\mathbb{Z}+\mathbb{Z} z), \lambda \alpha)
$$

for some $\lambda \in \mathbb{C}^{*}$, some $z \in \mathbb{H}$, and $\alpha \in M_{2}(\hat{\mathbb{Z}})$ (using the basis $(1,-z)$ of $\mathbb{Z}+\mathbb{Z} z$ as in (87) [10] to view $\alpha$ as a map $\phi)$. The diagonal action of $\Gamma=\mathrm{SL}_{2}(\mathbb{Z})$ yields isomorphic $\mathbb{Q}$-lattices, and $(c f$. (87) $[10])$ the space of 2 -dimensional $\mathbb{Q}$-lattices up to scaling can be identified with the quotient

$$
\Gamma \backslash\left(\mathrm{M}_{2}(\hat{\mathbb{Z}}) \times \mathbb{H}\right) .
$$

The relation of commensurability is implemented by the partially defined action of $\mathrm{GL}_{2}^{+}(\mathbb{Q})$ on $(3.35)$. We denote by $\mathcal{R}_{2}$ the groupoid of the commensurability relation on 2-dimensional $\mathbb{Q}$-lattices not up to scaling.

The groupoid $\mathcal{R}_{2}$ has, as (noncommutative) algebra of coordinates, the convolution algebra of $\Gamma \times \Gamma$ invariant functions on

$$
\tilde{\mathcal{U}}=\left\{(g, \alpha, u) \in \mathrm{GL}_{2}^{+}(\mathbb{Q}) \times M_{2}(\hat{\mathbb{Z}}) \times \mathrm{GL}_{2}^{+}(\mathbb{R}) \mid g \alpha \in M_{2}(\hat{\mathbb{Z}})\right\} .
$$

Up to Morita equivalence, this can also be described as the crossed product

$$
C_{0}\left(M_{2}\left(\mathbb{A}_{f}\right) \times \mathrm{GL}_{2}(\mathbb{R})\right) \rtimes \mathrm{GL}_{2}(\mathbb{Q}) .
$$

Passing to $\mathbb{Q}$-lattices up to scaling corresponds to taking the quotient

$$
Z:=\mathcal{R}_{2} / \mathbb{C}^{*}
$$

The action of $\mathbb{C}^{*}$ on $\mathbb{Q}$-lattices preserves the commensurability relation. However, the action is not free due to the presence of lattices $L=(0, z)$, where $z \in \Gamma \backslash \mathbb{H}$ has nontrivial automorphisms. Thus, the following problem arises.

Claim 3.4. The quotient $Z=\mathcal{R}_{2} / \mathbb{C}^{*}$ is no longer a groupoid.

Proof. This can be seen in the following simple example. Consider the two $\mathbb{Q}$-lattices $\left(\alpha_{1}, z_{1}\right)=$ $(0,2 \sqrt{-1})$ and $\left(\alpha_{2}, z_{2}\right)=(0, \sqrt{-1})$. The composite

$$
\left(\left(\alpha_{1}, z_{1}\right),\left(\alpha_{2}, z_{2}\right)\right) \circ\left(\left(\alpha_{2}, z_{2}\right),\left(\alpha_{1}, z_{1}\right)\right)
$$


is equal to the identity $\left(\left(\alpha_{1}, z_{1}\right),\left(\alpha_{1}, z_{1}\right)\right)$. We can also consider the composition

$$
\left(\sqrt{-1}\left(\alpha_{1}, z_{1}\right), \sqrt{-1}\left(\alpha_{2}, z_{2}\right)\right) \circ\left(\left(\alpha_{2}, z_{2}\right),\left(\alpha_{1}, z_{1}\right)\right),
$$

where $\sqrt{-1}\left(\alpha_{2}, z_{2}\right)=\left(\alpha_{2}, z_{2}\right)$, but this is not the identity, since $\sqrt{-1}\left(\alpha_{1}, z_{1}\right) \neq\left(\alpha_{1}, z_{1}\right)$.

However, we can still define a convolution algebra for the quotient $Z$ of (3.38), by restricting the convolution product of $\mathcal{R}_{2}$ to homogeneous functions of weight zero with $\mathbb{C}^{*}$-compact support, where a function $f$ has weight $k$ if it satisfies

$$
f(g, \alpha, u \lambda)=\lambda^{k} f(g, \alpha, u), \quad \forall \lambda \in \mathbb{C}^{*} .
$$

This is the analog of the description (3.9) for the 1-dimensional case.

Definition 3.5. The noncommutative algebra of coordinates $\mathcal{A}_{2}$ is the Hecke algebra of functions on

$$
\mathcal{U}=\left\{(g, \alpha, z) \in \mathrm{GL}_{2}^{+}(\mathbb{Q}) \times M_{2}(\hat{\mathbb{Z}}) \times \mathbb{H}, g \alpha \in M_{2}(\hat{\mathbb{Z}})\right\}
$$

invariant under the $\Gamma \times \Gamma$ action

$$
(g, \alpha, z) \mapsto\left(\gamma_{1} g \gamma_{2}^{-1}, \gamma_{2} \alpha, \gamma_{2}(z)\right),
$$

with convolution

$$
\left(f_{1} * f_{2}\right)(g, \alpha, z)=\sum_{s \in \Gamma \backslash \mathrm{GL}_{2}^{+}(\mathbb{Q}), s \alpha \in M_{2}(\hat{\mathbb{Z}})} f_{1}\left(g s^{-1}, s \alpha, s(z)\right) f_{2}(s, \alpha, z)
$$

and adjoint $f^{*}(g, \alpha, z)=\overline{f\left(g^{-1}, g \alpha, g(z)\right)}$.

This contains the classical Hecke operators (cf. (128) [10]). The time evolution determined by the ratio of covolumes of pairs of commensurable $\mathbb{Q}$-lattices is given by

$$
\sigma_{t}(f)(g, \alpha, \tau)=\operatorname{det}(g)^{i t} f(g, \alpha, \tau)
$$

where, for the pair of commensurable $\mathbb{Q}$-lattices associated to $(g, \alpha, \tau)$, one has

$$
\operatorname{det}(g)=\operatorname{covolume}\left(\Lambda^{\prime}\right) / \operatorname{covolume}(\Lambda) .
$$

\subsection{Tate modules and Shimura varieties.}

We now give a description closer to (3.13), which shows that again we can interpret the space of commensurability classes of 2-dimensional $\mathbb{Q}$-lattices up to scaling as a noncommutative version of the Shimura variety (3.33). More precisely, we give a reinterpretation of the notion of 2-dimensional $\mathbb{Q}$-lattices and commensurability, which may be useful in the context of similar quantum statistical mechanical systems associated to more general Shimura varieties as in [13].

Implicit in what follows is an isomorphism between $\mathbb{Q} / \mathbb{Z}$ and the roots of unity in $\mathbb{C}$. For instance, this can be given by the exponential function $e^{2 \pi i z}$.

Proposition 3.6. The data of a 2-dimensional $\mathbb{Q}$-lattice up to scaling are equivalent to the data of an elliptic curve $E$, together with a pair of points $\xi=\left(\xi_{1}, \xi_{2}\right)$ in the cohomology $H^{1}(E, \hat{\mathbb{Z}})$. Commensurability of 2-dimensional $\mathbb{Q}$-lattices up to scale is then implemented by an isogeny of the corresponding elliptic curves, with the elements $\xi$ and $\xi^{\prime}$ related via the induced map in cohomology.

Proof. The subgroup $\mathbb{Q} \Lambda / \Lambda$ of $\mathbb{C} / \Lambda=E$ is the torsion subgroup $E_{\text {tor }}$ of the elliptic curve $E$. Thus, one can rewrite the map $\phi$ as a map $\mathbb{Q}^{2} / \mathbb{Z}^{2} \rightarrow E_{\text {tor }}$. Using the canonical isomorphism $E[n] \stackrel{\sim}{\longrightarrow}$ $H^{1}(E, \mathbb{Z} / n \mathbb{Z})$, for $E[n]=\Lambda / n \Lambda$ the $n$-torsion points of $E$, one can interpret $\phi$ as a map $\mathbb{Q}^{2} / \mathbb{Z}^{2} \rightarrow$ $H^{1}(E, \mathbb{Q} / \mathbb{Z})$.

By taking $\operatorname{Hom}(\mathbb{Q} / \mathbb{Z},-)$, the map $\phi$ corresponds to a $\hat{\mathbb{Z}}$-linear map

$$
\hat{\mathbb{Z}} \oplus \hat{\mathbb{Z}} \rightarrow H^{1}(E, \hat{\mathbb{Z}})
$$

or to a choice of two elements of the latter. In fact, we use here the identification

$$
H^{1}(E, \hat{\mathbb{Z}}) \cong T E
$$


of $H^{1}(E, \hat{\mathbb{Z}})=H^{1}(E, \mathbb{Z}) \otimes_{\mathbb{Z}} \hat{\mathbb{Z}}$ with the total Tate module

$$
\Lambda \otimes \hat{\mathbb{Z}}=\underbrace{\lim }_{n} E[n]=T E .
$$

Thus, (3.44) gives a cohomological formulation of the $\hat{\mathbb{Z}}$-linear map $\phi: \hat{\mathbb{Z}} \oplus \hat{\mathbb{Z}} \rightarrow \Lambda \otimes \hat{\mathbb{Z}}$. Commensurability of $\mathbb{Q}$-lattices up to scale is rephrased as the condition that the elliptic curves are isogenous and the points in the Tate module are related via the induced map in cohomology.

Another reformulation uses the Pontrjagin duality between profinite abelian groups and discrete torsion abelian groups given by $\operatorname{Hom}(-, \mathbb{Q} / \mathbb{Z})$. This reformulates the datum $\phi$ of a $\mathbb{Q}$-lattice as a $\hat{\mathbb{Z}}$-linear $\operatorname{map} \operatorname{Hom}(\mathbb{Q} \Lambda / \Lambda, \mathbb{Q} / \mathbb{Z}) \rightarrow \hat{\mathbb{Z}} \oplus \hat{\mathbb{Z}}$, which is identified with $\Lambda \otimes \hat{\mathbb{Z}} \rightarrow \hat{\mathbb{Z}} \oplus \hat{\mathbb{Z}}$. Here we use the fact that $\Lambda$ and $\Lambda \otimes \hat{\mathbb{Z}} \cong H^{1}(E, \hat{\mathbb{Z}})$ are both self-dual (Poincaré duality of $E$ ). In this dual formulation commensurability means that the two maps agree on the intersection of the two commensurable lattices, $\left(\Lambda_{1} \cap \Lambda_{2}\right) \otimes \hat{\mathbb{Z}}$.

With the formulation of Proposition 3.6, we can then give a new interpretation of the result of Proposition 43 of [10], which shows that the space of commensurability classes of 2-dimensional $\mathbb{Q}$ lattices up to scaling is described by the quotient

$$
S h^{(n c)}\left(\mathbb{H}^{ \pm}, \mathrm{GL}_{2}\right):=\mathrm{GL}_{2}(\mathbb{Q}) \backslash\left(M_{2}\left(\mathbb{A}_{f}\right) \times \mathbb{H}^{ \pm}\right) .
$$

In fact, commensurability classes of $\mathbb{Q}$-lattices $(\Lambda, \phi)$ in $\mathbb{C}$ are the same as isogeny classes of data $(E, \eta)$ of elliptic curves $E=\mathbb{C} / \Lambda$ and $\mathbb{A}_{f}$-homomorphisms

$$
\eta: \mathbb{Q}^{2} \otimes \mathbb{A}_{f} \rightarrow \Lambda \otimes \mathbb{A}_{f}
$$

with $\Lambda \otimes \mathbb{A}_{f}=(\Lambda \otimes \hat{\mathbb{Z}}) \otimes \mathbb{Q}$, where we can identify $\Lambda \otimes \hat{\mathbb{Z}}$ with the total Tate module of $E$, as in (3.45). Since the $\mathbb{Q}$-lattice need not be invertible, we do not require that $\eta$ be an $\mathbb{A}_{f}$-isomorphism $(c f$. [25]). In fact, the commensurability relation between $\mathbb{Q}$-lattices corresponds to the equivalence $(E, \eta) \sim$ $\left(E^{\prime}, \eta^{\prime}\right)$ given by an isogeny $g: E \rightarrow E^{\prime}$ and $\eta^{\prime}=(g \otimes 1) \circ \eta$. Namely, the equivalence classes can be identified with the quotient of $M_{2}\left(\mathbb{A}_{f}\right) \times \mathbb{H}^{ \pm}$by the action of $\mathrm{GL}_{2}(\mathbb{Q}),(\rho, z) \mapsto(g \rho, g(z))$.

Thus, (3.46) describes a noncommutative Shimura variety which has the Shimura variety (3.33) as the set of its classical points. The results of [10] show that, as in the case of the BC system, the set of low temperature extremal KMS states is a classical Shimura variety. We shall return to this in the next section.

Remark 3.7. Under the Gelfand-Naimark correspondence, unital $C^{*}$-algebras correspond to compact spaces. Thus, one can say that a noncommutative space $X$ described by a $C^{*}$-algebra $\mathcal{A}$ is "Moritacompact" if the algebra $\mathcal{A}$ is Morita equivalent to a unital $C^{*}$-algebra.

This provides us with a notion of "Morita-compactification" for noncommutative spaces. In the case of the $\mathrm{GL}_{2}$-system, we can replace (3.46) by the noncommutative space

$$
\overline{S h^{(n c)}}\left(\mathbb{H}^{ \pm}, \mathrm{GL}_{2}\right):=\mathrm{GL}_{2}(\mathbb{Q}) \backslash\left(M_{2}\left(\mathbb{A}_{f}\right) \times \mathbb{P}^{1}(\mathbb{C})\right) \sim \mathrm{GL}_{2}(\mathbb{Q}) \backslash M_{2}(\mathbb{A})^{\cdot} / \mathbb{C}^{*},
$$

where we use the notation $M_{2}(\mathbb{A})^{\cdot}=M_{2}\left(\mathbb{A}_{f}\right) \times\left(M_{2}(\mathbb{R}) \backslash\{0\}\right)$. This corresponds to allowing degenerations of the underlying lattice in $\mathbb{C}$ to a pseudolattice $(c f .[22])$, while maintaining the $\mathbb{Q}$-structure (cf. [10]). Thus, the space (3.48) can be thought of as obtained from the classical Shimura variety $S h\left(\mathrm{GL}_{2}, \mathbb{H}^{ \pm}\right)$by allowing all possible degenerations of the lattice, both at the archimedean and at the non-archimedean components. In the archimedan case, this accounts for adding the rank one matrices to $G L_{2}(\mathbb{R})$, so as to obtain $M_{2}(\mathbb{R}) \backslash\{0\} / \mathbb{C}^{*}=\mathbb{P}^{1}(\mathbb{R})$, which corresponds to the pseudo-lattices. In the non-archimedean part one allows the presence of non-invertible $\mathbb{Q}$-lattices, which, by Proposition 3.6, can also be viewed (adelically) as an analogous degenerations (the two points $\xi_{i}$ in the Tate module need not be independent). The "invertible part"

$$
\mathrm{GL}_{2}(\mathbb{Q}) \backslash\left(\mathrm{GL}_{2}\left(\mathbb{A}_{f}\right) \times \mathbb{P}^{1}(\mathbb{R})\right)
$$


of the "boundary" gives the noncommutative modular tower considered in [9], [24], and [29], so that the full space (3.48) appears to be the most natural candidate for the geometry underlying the construction of a quantum statistical mechanical system adapted to the case of both imaginary and real quadratic fields (cf. [22] [23]).

\subsection{Arithmetic properties of the $\mathrm{GL}_{2}$-system.}

The result proved in [10] for the $\mathrm{GL}_{2}$-system shows that the action of symmetries on the extremal KMS states at zero temperature is now related to the Galois theory of the field of modular functions.

Since the arithmetic subalgebra for the BC system was obtained by considering weight zero lattice functions of the form (3.18), it is natural to expect that the analog for the $\mathrm{GL}_{2}$-system will involve lattice functions given by the Eisenstein series, suitably normalized to weight zero, according to the analogy developed by Kronecker between trigonometric and elliptic functions, as outlined by A.Weil in [34]. This suggests that modular functions should appear naturally in the arithmetic subalgebra $\mathcal{A}_{2, \mathbb{Q}}$ of the $\mathrm{GL}_{2}$-system, but that requires working with unbounded multipliers.

This is indeed the case for the arithmetic subalgebra $\mathcal{A}_{2, \mathbb{Q}}$ defined in [10], which we now recall. The main point of the following definition is that the rationl subalgebra of the $\mathrm{GL}_{2}$-system is obtained by assigning the simplest condition of algebraicity on the parameters the algebra depends on.

The $q$-expansion at a cusp of modular functions of level $N$ in the modular field $F$ ( $c f$. Section 1.1) has coefficients in the cyclotomic field $\mathbb{Q}\left(\zeta_{N}\right)$. Thus, the Galois group $\operatorname{Gal}\left(\mathbb{Q}^{a b} / \mathbb{Q}\right)$ acts on these coefficients through $\operatorname{Gal}\left(\mathbb{Q}\left(\zeta_{N}\right) / \mathbb{Q}\right)$. This action of $\hat{\mathbb{Z}}^{*} \simeq \operatorname{Gal}\left(\mathbb{Q}^{a b} / \mathbb{Q}\right)$ on the coefficients of the $q$ expansion determines a homomorphism

$$
\operatorname{cycl}: \hat{\mathbb{Z}}^{*} \rightarrow \operatorname{Aut}(F) \text {. }
$$

For $\rho \in \hat{\mathbb{Z}}^{*}$, this sends a modular function $f \in F$ with coefficients $a_{n}$ of the $q$-expansion to the modular function $\operatorname{cycl}(\rho)(f)$ that has the $\rho\left(a_{n}\right)$ as coefficients.

If $f$ is a continuous function on the quotient $Z$ of (3.38), we write

$$
f_{(g, \alpha)}(z)=f(g, \alpha, z)
$$

so that $f_{(g, \alpha)} \in C(\mathbb{H})$. Here the coordinates $(g, \alpha, z)$ are as in $(3.39)$, with $z \in \mathrm{GL}_{2}^{+}(\mathbb{R}) / \mathbb{C}^{*}$.

For $p_{N}: M_{2}(\hat{\mathbb{Z}}) \rightarrow M_{2}(\mathbb{Z} / N \mathbb{Z})$ the canonical projection, we say that $f$ is of level $N$ if

$$
f_{(g, \alpha)}=f_{\left(g, p_{N}(\alpha)\right)} \quad \forall(g, \alpha) .
$$

Then $f$ is completely determined by the functions

$$
f_{(g, m)} \in C(\mathbb{H}), \quad \text { for } m \in M_{2}(\mathbb{Z} / N \mathbb{Z})
$$

Definition 3.8. [10] The arithmetic algebra $\mathcal{A}_{2, \mathbb{Q}}$ is a subalgebra of continuous functions on the quotient $Z$ of (3.38), with the convolution product (3.41) and with the properties:

- The support of $f$ in $\Gamma \backslash \mathrm{GL}_{2}^{+}(\mathbb{Q})$ is finite.

- The function $f$ is of finite level.

- For all $(g, m)$, the function $f$ satisfies $f_{(g, m)} \in F$.

- The function $f$ satisfies the cyclotomic condition:

$$
f_{(g, \alpha(u) m)}=\operatorname{cycl}(u) f_{(g, m)},
$$

for all $g \in \mathrm{GL}_{2}^{+}(\mathbb{Q})$ diagonal and all $u \in \hat{\mathbb{Z}}^{*}$, with

$$
\alpha(u)=\left(\begin{array}{ll}
u & 0 \\
0 & 1
\end{array}\right)
$$

and cycl as in (3.50). 
Notice that the invariance $f(g \gamma, \alpha, z)=f(g, \gamma \alpha, \gamma(z))$, for all $\gamma \in \Gamma$ and for all $(g, \alpha, z) \in \mathcal{U}$, implies that $f_{(g, m) \mid \gamma}=f_{(g, m)}$, for all $\gamma \in \Gamma(N) \cap g^{-1} \Gamma g$, i.e. $f$ is invariant under a congruence subgroup.

The cyclotomic condition is a consistency condition on the roots of unity that appear in the coefficients of the $q$-series. It only depends on the simple action of $\hat{\mathbb{Z}}^{*}$ on the coefficients, but it is crucial for the existence of "fabulous states" for the action of the Galois group of the modular field ( $c f .[10])$.

It is important to remark here, for the later application to the case of imaginary quadratic fields, that the Galois theory of the modular field is not built into the definition of the arithmetic algebra $\mathcal{A}_{2, \mathbb{Q}}$. In fact, the properties we assume in Definition 3.8 arise by just requiring a natural algebraicity condition on the only continuous modulus $q$ present in the algebra (while eliminating the trivial cases through the cyclotomic condition). The refined theory of automorphisms of the modular field does not enter in any way in the definition of the algebra.

For $\alpha \in M_{2}(\hat{\mathbb{Z}})$, let $G_{\alpha} \subset \mathrm{GL}_{2}^{+}(\mathbb{Q})$ be the set of

$$
G_{\alpha}=\left\{g \in \mathrm{GL}_{2}^{+}(\mathbb{Q}): g \alpha \in M_{2}(\hat{\mathbb{Z}})\right\} .
$$

Then, as shown in [10], an element $y=(\alpha, z) \in M_{2}(\hat{\mathbb{Z}}) \times \mathbb{H}$ determines a unitary representation of the Hecke algebra $\mathcal{A}$ on the Hilbert space $\ell^{2}\left(\Gamma \backslash G_{\alpha}\right)$,

$$
\left(\left(\pi_{y} f\right) \xi\right)(g):=\sum_{s \in \Gamma \backslash G_{\alpha}} f\left(g s^{-1}, s \alpha, s(z)\right) \xi(s), \quad \forall g \in G_{\alpha}
$$

for $f \in \mathcal{A}$ and $\xi \in \ell^{2}\left(\Gamma \backslash G_{\alpha}\right)$.

Invertible $\mathbb{Q}$-lattices determine positive energy representations, due to the fact that the condition $g \alpha \in M_{2}(\hat{\mathbb{Z}})$ for $g \in \mathrm{GL}_{2}^{+}(\mathbb{Q})$ and $\alpha \in \mathrm{GL}_{2}(\hat{\mathbb{Z}})$ (invertible case) implies $g \in M_{2}(\mathbb{Z})^{+}$, hence the time evolution (3.42) is implemented by the positive Hamiltonian with spectrum $\{\log \operatorname{det}(m)\} \subset[0, \infty)$ for $m \in \Gamma \backslash M_{2}(\mathbb{Z})^{+}$. The partition function of the $\mathrm{GL}_{2}$-system is then $Z(\beta)=\zeta(\beta) \zeta(\beta-1)$. This shows that one can expect the system to have two phase transitions, which is in fact the case.

While the group $\mathrm{GL}_{2}(\hat{\mathbb{Z}})$ acts by automorphisms of the algebra of coordinates of $\mathcal{R}_{2} / \mathbb{C}^{*}$, i.e. the algebra of the quotient (3.46), the action of $\mathrm{GL}_{2}\left(\mathbb{A}_{f}\right)$ on the Hecke algebra $\mathcal{A}_{2}$ of coordinates of $\mathcal{R}_{2} / \mathbb{C}^{*}$ is by endomorphisms. More precisely, the group $\mathrm{GL}_{2}(\hat{\mathbb{Z}})$ of deck transformations of the modular tower still acts by automorphisms on this algebra, while $\mathrm{GL}_{2}^{+}(\mathbb{Q})$ acts by endomorphisms of the $C^{*}$-dynamical system, with the diagonal $\mathbb{Q}^{*}$ acting by inner, as in (2.5).

The group of symmetries $\mathrm{GL}_{2}\left(\mathbb{A}_{f}\right)$ preserves the arithmetic subalgebra; we remark that $\mathrm{GL}_{2}\left(\mathbb{A}_{f}\right)=$ $\mathrm{GL}_{2}^{+}(\mathbb{Q}) \cdot \mathrm{GL}_{2}(\hat{\mathbb{Z}})$. In fact, the group $\mathbb{Q}^{*} \backslash \mathrm{GL}_{2}\left(\mathbb{A}_{f}\right)$ has an important arithmetic meaning: a result of Shimura (cf. [32], [21]) characterizes the automorphisms of the modular field by the exact sequence

$$
0 \rightarrow \mathbb{Q}^{*} \rightarrow \mathrm{GL}_{2}\left(\mathbb{A}_{f}\right) \rightarrow \operatorname{Aut}(F) \rightarrow 0 .
$$

There is an induced action of $\mathbb{Q}^{*} \backslash \mathrm{GL}_{2}\left(\mathbb{A}_{f}\right)$ (symmetries modulo inner) on the $\mathrm{KMS}_{\beta}$ states of $\left(\mathcal{A}, \sigma_{t}\right)$, for $\beta<\infty$. The action of $\mathrm{GL}_{2}(\hat{\mathbb{Z}})$ extends to $\mathrm{KMS}_{\infty}$ states, while the action of $\mathrm{GL}_{2}^{+}(\mathbb{Q})$ on $\Sigma_{\infty}$ is defined by the action at finite (large) $\beta$, by first "warming up" and then "cooling down" as in (2.7) $(c f .[10])$.

The result of [10] on the structure of KMS states for the $\mathrm{GL}_{2}$ system is as follows.

Theorem 3.9. (Connes-Marcolli [10]) For the system $\left(\mathcal{A}_{2}, \sigma_{t}\right)$ described above, the structure of the KMS states is as follows.

- There is no KMS state in the range $0<\beta \leq 1$.

- In the range $\beta>2$ the set of extremal KMS states is given by the invertible $\mathbb{Q}$-lattices, namely by the Shimura variety $S h\left(\mathrm{GL}_{2}, \mathbb{H}^{ \pm}\right)$,

$$
\mathcal{E}_{\beta} \cong \mathrm{GL}_{2}(\mathbb{Q}) \backslash \mathrm{GL}_{2}(\mathbb{A}) / \mathbb{C}^{*} .
$$


The explicit expression for these extremal $K M S_{\beta}$ states is

$$
\varphi_{\beta, L}(f)=\frac{1}{Z(\beta)} \sum_{m \in \Gamma \backslash M_{2}^{+}(\mathbb{Z})} f(1, m \alpha, m(z)) \operatorname{det}(m)^{-\beta}
$$

where $L=(\alpha, z)$ is an invertible $\mathbb{Q}$-lattice.

- At $\beta=\infty$, and for generic $L=(\alpha, \tau)$ invertible (where generic means $j(\tau) \notin \overline{\mathbb{Q}})$, the values of the state $\varphi_{\infty, L} \in \mathcal{E}_{\infty}$ on elements of $\mathcal{A}_{2, \mathbb{Q}}$ lie in an embedded image in $\mathbb{C}$ of the modular field,

and there is an isomorphism

$$
\theta_{\varphi}: \operatorname{Aut}_{\mathbb{Q}}\left(F_{\tau}\right) \stackrel{\simeq}{\longrightarrow} \mathbb{Q}^{*} \backslash \mathrm{GL}_{2}\left(\mathbb{A}_{f}\right),
$$

depending on $L=(\alpha, \tau)$, which intertwines the Galois action on the values of the state with the action of symmetries,

$$
\gamma \varphi(f)=\varphi\left(\theta_{\varphi}(\gamma) f\right), \quad \forall f \in \mathcal{A}_{2, \mathbb{Q}}, \quad \forall \gamma \in \operatorname{Aut}_{\mathbb{Q}}\left(F_{\tau}\right)
$$

\subsection{Crossed products and functoriality.}

In the case of the classical Shimura varieties, the relation between (3.33) and (3.4) is given by "passing to components", namely we have $(c f .[25])$

$$
\pi_{0}\left(S h\left(\mathrm{GL}_{2}, \mathbb{H}^{ \pm}\right)\right)=S h\left(\mathrm{GL}_{1},\{ \pm 1\}\right) .
$$

In fact, the operation of taking connected components of (3.33) is realized by the map

$$
\operatorname{sign} \times \operatorname{det}: S h\left(\mathrm{GL}_{2}, \mathbb{H}^{ \pm}\right) \rightarrow S h\left(\mathrm{GL}_{1},\{ \pm 1\}\right) .
$$

We show here in Proposition 3.10 that this compatibility extends to a correspondence between the noncommutative Shimura varieties (3.14) and (3.46), compatible with the time evolutions and the arithmetic structures.

We have seen that, up to Morita equivalence, the noncommutative spaces of $\mathbb{Q}$-lattices modulo commensurability (but not scaling) in dimensions $n=1,2$ are described, respectively, by the crossed product $C^{*}$-algebras

$$
C_{0}\left(\mathbb{A}_{f} \times \mathrm{GL}_{1}(\mathbb{R})\right) \rtimes \mathrm{GL}_{1}(\mathbb{Q}),
$$

and

$$
C_{0}\left(M_{2}\left(\mathbb{A}_{f}\right) \times \mathrm{GL}_{2}(\mathbb{R})\right) \rtimes \mathrm{GL}_{2}(\mathbb{Q}) .
$$

The functor $C_{0}$ from locally compact spaces to $C^{*}$-algebras is contravariant, while the functor $C^{*}$ from discrete groups to the associated (maximal) convolution $C^{*}$-algebra is covariant. This shows that one needs to be careful in getting functoriality for crossed products.

Thus, in order to get a morphism of crossed product algebras

$$
C_{0}\left(X_{1}\right) \rtimes \Gamma_{1} \rightarrow C_{0}\left(X_{2}\right) \rtimes \Gamma_{2}
$$

it is necessary to have a proper continuous map $\pi: X_{2} \rightarrow X_{1}$ and a proper group homomorphism $\alpha: \Gamma_{1} \rightarrow \Gamma_{2}$, such that

$$
\pi \circ \alpha(g)=g \circ \pi \quad \forall g \in \Gamma_{1} .
$$

In this case, one obtains an algebra homomorphism

$$
\sum f_{g} U_{g} \in C_{0}\left(X_{1}\right) \rtimes \Gamma_{1} \mapsto \sum\left(f_{g} \circ \pi\right) U_{\alpha(g)} \in C_{0}\left(X_{2}\right) \rtimes \Gamma_{2} .
$$

When the continuous map $\pi$ fails to be proper, the above formula only defines a homomorphism to the multiplier algebra

$$
C_{0}\left(X_{1}\right) \rtimes \Gamma_{1} \rightarrow M\left(C_{0}\left(X_{2}\right) \rtimes \Gamma_{2}\right)
$$


In the case of the $\mathrm{BC}$ and the $\mathrm{GL}_{2}$ systems, we consider the map $\alpha: \mathrm{GL}_{1}(\mathbb{Q}) \rightarrow \mathrm{GL}_{2}(\mathbb{Q})$ of the form

$$
\alpha(r)=\left(\begin{array}{ll}
r & 0 \\
0 & 1
\end{array}\right)
$$

We also take $\pi$ to be the determinant map,

$$
(\rho, u) \in M_{2}\left(\mathbb{A}_{f}\right) \times \mathrm{GL}_{2}(\mathbb{R}) \mapsto \pi(\rho, u)=(\operatorname{det}(\rho), \operatorname{det}(u)) \in \mathbb{A}_{f} \times \mathrm{GL}_{1}(\mathbb{R}) .
$$

We then have the following result extending the classical map of Shimura varieties (3.58).

Proposition 3.10. Let $\alpha$ and $\pi$ be defined as in (3.64) and (3.65). Then the following holds.

(1) The pair $(\pi, \alpha)$ induces a morphism

$$
\rho: C_{0}\left(\mathbb{A}_{f} \times \mathrm{GL}_{1}(\mathbb{R})\right) \rtimes \mathrm{GL}_{1}(\mathbb{Q}) \rightarrow M\left(C_{0}\left(M_{2}\left(\mathbb{A}_{f}\right) \times \mathrm{GL}_{2}(\mathbb{R})\right) \rtimes \mathrm{GL}_{2}(\mathbb{Q})\right) .
$$

(2) The restriction of $\rho$ to homogeneous functions of weight 0 induces a morphism

$$
\rho_{0}: C_{0}\left(\mathbb{A}_{f} \times\{ \pm 1\}\right) \rtimes \mathrm{GL}_{1}(\mathbb{Q}) \rightarrow M\left(C_{0}\left(M_{2}\left(\mathbb{A}_{f}\right) \times \mathbb{H}^{ \pm}\right) \rtimes \mathrm{GL}_{2}(\mathbb{Q})\right) .
$$

(3) The above yields a correspondence $\rho_{12}$ from the $\mathrm{GL}_{1}$-system to the $\mathrm{GL}_{2}$-system, compatible with the time evolutions and the rational subalgebras.

Proof. 1) One checks that (3.62) holds, since $\alpha$ satisfies $\operatorname{det} \alpha(r)=r$. By construction, $\pi$ is not proper but is clearly continuous, hence one obtains the morphism $\rho$ to the multiplier algebra, as we discussed above.

2) The extension $\tilde{\rho}$ of $\rho$ to multipliers gives a morphism

$$
\tilde{\rho}: C\left(\mathbb{A}_{f} \times \mathrm{GL}_{1}(\mathbb{R})\right) \rtimes \mathrm{GL}_{1}(\mathbb{Q}) \rightarrow C\left(M_{2}\left(\mathbb{A}_{f}\right) \times \mathrm{GL}_{2}(\mathbb{R})\right) \rtimes \mathrm{GL}_{2}(\mathbb{Q}) .
$$

One obtains $\rho_{0}$ by restricting $\tilde{\rho}$ to homogeneous functions of weight 0 for the scaling action of $\mathbb{R}_{+}^{*}$ on $\mathrm{GL}_{1}(\mathbb{R})$. The image of such a function is homogeneous of weight 0 for the scaling action of $\mathbb{C}^{*}$ on $\mathrm{GL}_{2}(\mathbb{R})$ and only depends upon the sign of the modulus in $\mathbb{H}^{ \pm}$as in the diagram

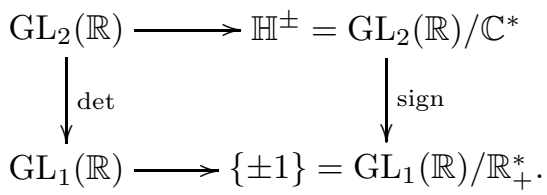

3) One can restrict $\rho_{0}$ to $\mathcal{A}_{1}=C(\hat{\mathbb{Z}}) \rtimes_{\alpha} \mathbb{N}^{\times}$and one gets a morphism

$$
C(\hat{\mathbb{Z}}) \rtimes_{\alpha} \mathbb{N}^{\times} \rightarrow M\left(C_{0}\left(M_{2}\left(\mathbb{A}_{f}\right) \times \mathbb{H}\right) \rtimes \mathrm{GL}_{2}(\mathbb{Q})^{+},\right)
$$

but one still needs to combine it with the Morita equivalence between $C_{0}\left(M_{2}\left(\mathbb{A}_{f}\right) \times \mathbb{H}\right) \rtimes \mathrm{GL} 2(\mathbb{Q})^{+}$ and $\mathcal{A}_{2}$. This Morita equivalence is given by the bimodule $\mathcal{E}$ of functions of

$$
(g, \alpha, z) \in \mathcal{V}=\mathrm{GL}_{2}^{+}(\mathbb{Q}) \times M_{2}(\hat{\mathbb{Z}}) \times \mathbb{H}
$$

invariant under the $\Gamma$ action

$$
(g, \alpha, z) \mapsto\left(g \gamma^{-1}, \gamma \alpha, \gamma(z)\right) .
$$

For $(g, \alpha, z) \in \mathcal{V}$, both $g \alpha \in M_{2}\left(\mathbb{A}_{f}\right)$ and $g(z) \in \mathbb{H}$ make sense, and this allows for a left action of $C_{0}\left(M_{2}\left(\mathbb{A}_{f}\right) \times \mathbb{H}\right)$ on $\mathcal{E}$. This action is $\mathrm{GL}_{2}^{+}(\mathbb{Q})$-equivariant for the left action of $\mathrm{GL}_{2}^{+}(\mathbb{Q})$ on $\mathcal{V}$ given by

$$
h(g, \alpha, z)=(h g, \alpha, z) \quad \forall h \in \mathrm{GL}_{2}^{+}(\mathbb{Q})
$$

and turns $\mathcal{E}$ into a left $C_{0}\left(M_{2}\left(\mathbb{A}_{f}\right) \times \mathbb{H}\right) \rtimes \mathrm{GL}_{2}(\mathbb{Q})^{+}$module. To obtain the right module structure over $\mathcal{A}_{2}$, one uses the convolution product

$$
(\xi * f)(g, \alpha, z)=\sum_{s \in \Gamma \backslash \mathrm{GL}_{2}^{+}(\mathbb{Q})} \xi\left(g s^{-1}, s \alpha, s(z)\right) f(s, \alpha, z) .
$$


The left action of $C_{0}\left(M_{2}\left(\mathbb{A}_{f}\right) \times \mathbb{H}\right)$ extends to its multipliers and one can use (3.69) to obtain on $\mathcal{E}$ the required structure of left $C(\hat{\mathbb{Z}}) \rtimes_{\alpha} \mathbb{N}^{\times}$-module and right $\mathcal{A}_{2}$-module. One checks that this is compatible with the time evolutions $\sigma_{t}$. It is also compatible with the rational subalgebras $\mathcal{A}_{1, \mathbb{Q}}$ and $\mathcal{A}_{2, \mathbb{Q}}$. This compatibility can be seen using the cyclotomic condition defined in (3.50) and in Definition 3.8.

The compatibility between the BC system and the CM system for imaginary quadratic fields is discussed in Proposition 4.9.

\section{Quantum STATISTICAL MEChanics FOR IMAGinaRY QUADRATIC FIELDS}

In the Kronecker-Weber case, the maximal abelian extension of $\mathbb{Q}$ is generated by the values of the exponential function at the torsion points $\mathbb{Q} / \mathbb{Z}$ of the group $\mathbb{C} / \mathbb{Z}=\mathbb{C}^{*}$.

Similarly, it is well known that the maximal abelian extension of an imaginary quadratic field $K$ is generated by the values of a certain analytic function, the Weierstrass $\wp$-function, at the torsion points $E_{\text {tors }}$ of an elliptic curve $E$ (with complex multiplication by $\mathcal{O}$ ). It contains the $j$-invariant $j(E)$ of $E$. To see this, let $e_{k}$, for $k=1,2,3$, denote the three values taken by the Weierstrass $\wp$-function on the set $E[2]$ of 2-torsion points of the elliptic curve $E$. Then $j(E)$ is obtained as a function of the $e_{k}$ by the formula $j(E)=256\left(1-f+f^{2}\right)^{3} /\left(f^{2}(1-f)^{2}\right)$, where $f=\left(e_{2}-e_{3}\right) /\left(e_{1}-e_{3}\right)$.

Thus, in the case of imaginary quadratic fields, the theory of complex multiplication of elliptic curves provides a description of $K^{a b}$. The ideal class group $\mathrm{Cl}(\mathcal{O})$ is naturally isomorphic to $\operatorname{Gal}(K(j) / K)$, where $K(j(E))$ is the Hilbert class field of $K$, i.e. , its maximal abelian unramified extension. In the case that $\mathrm{Cl}(\mathcal{O})$ is trivial, the situation of the $\mathrm{CM}$ case is exactly as for the field $\mathbb{Q}$, with $\hat{\mathcal{O}}^{*}$ replacing $\hat{\mathbb{Z}}^{*}$. Namely, in the case of class number one, the class field theory isomorphism reduces to an isomorphism $\operatorname{Gal}\left(K^{a b} / K\right) \cong \hat{\mathcal{O}}^{*} / \mathcal{O}^{*}$, which is a direct generalization of $\operatorname{Gal}\left(\mathbb{Q}^{a b} / \mathbb{Q}\right) \cong \hat{\mathbb{Z}}^{*}$.

The construction of $\mathrm{BC}[3]$ was partially generalized to other global fields (i.e. number fields and function fields) in $[20,16,5]$. The construction of [16] involves replacing the ring $\mathcal{O}$ of integers of $K$ by a localized ring $\mathcal{O}_{S}$ which is principal and then taking a cross product of the form

$$
C^{*}\left(K / \mathcal{O}_{S}\right) \rtimes \mathcal{O}_{+}^{\times}
$$

where $\mathcal{O}_{+}^{\times}$is the sub semi-group of $K^{*}$ generated by the generators of prime ideals of $\mathcal{O}_{S}$. The symmetry group is $\hat{\mathcal{O}}_{S}^{*}$ and does not coincide with what is needed for class field theory except when the class number is 1 . The construction of [5] involves a cross product of the form

$$
C^{*}(K / \mathcal{O}) \rtimes J^{+}
$$

where $J^{+}$is a suitable adelic lift of the quotient group $\mathbb{I}_{K} / \hat{\mathcal{O}}^{*}$. It gives the right partition function namely the Dedekind zeta function but not the expected symmetries. The construction of [20] involves the algebra

$$
C^{*}(K / \mathcal{O}) \rtimes \mathcal{O}^{\times} \cong C(\hat{\mathcal{O}}) \rtimes \mathcal{O}^{\times}
$$

and has symmetry group $\hat{\mathcal{O}}^{*}$, while what is needed for class field theory is a system with symmetry group $\mathbb{I}_{K} / K^{*}$. As one can see from the commutative diagram

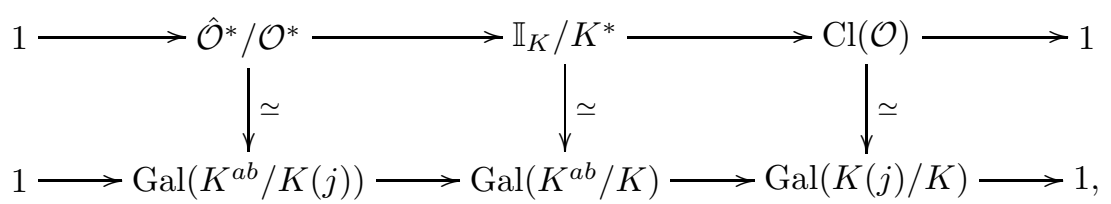

the action of $\hat{\mathcal{O}}^{*}$ is sufficient only in the case when the class number is one. In order to avoid the class number one restriction in extending the results of [3] to imaginary quadratic fields, it is natural to consider the universal situation: the moduli space of elliptic curves with level structure, i.e., the modular tower. Using the generalization of the $\mathrm{BC}$ case to $\mathrm{GL}_{2}$ constructed in [10], we will now describe the CM system constructed in [11]. This does in fact have the right properties to recover the 
explicit class field theory of imaginary quadratic fields from KMS states. It is based on the geometric notions of $K$-lattice and commensurability and extends to quadratic fields the reinterpretation of the BC system which was given in $[10]$ in terms of $\mathbb{Q}$-lattices. We show in this section that the CM system we obtained in [11] is, in fact, Morita equivalent to the one of [20]. The two main new ingredients in our construction are the choice of a natural rational subalgebra on which to evaluate the $\mathrm{KMS}_{\infty}$ states and the fact that the group of automorphisms $\hat{\mathcal{O}}^{*} / \mathcal{O}^{*}$ should be enriched by further symmetries, this time given by endomorphisms, so that the actual group of symmetries of the system is exactly $\mathbb{I}_{K} / K^{*}$. In particular, the choice of the rational subalgebra differs from [20], hence, even though Morita equivalent, the systems are inequivalent as "noncommutative pro-varieties over $\mathbb{Q}$ ".

\section{1. $K$-lattices and commensurability.}

In order to compare the $\mathrm{BC}$ system, the $\mathrm{GL}_{2}$ system and the $\mathrm{CM}$ case, we give a definition of $K$ lattices, for $K$ an imaginary quadratic field. The quantum statistical mechanical system we shall construct to recover the explicit class field theory of imaginary quadratic fields will be related to commensurability of 1-dimensional $K$-lattices. This will be analogous to the description of the BC system in terms of commensurability of 1 -dimensional $\mathbb{Q}$-lattices. On the other hand, since there is a forgetful map from 1-dimensional $K$-lattices to 2 -dimensional $\mathbb{Q}$-lattices, we will also be able to treat the $\mathrm{CM}$ case as a specialization of the $\mathrm{GL}_{2}$ system at $\mathrm{CM}$ points.

Let $\mathcal{O}=\mathbb{Z}+\mathbb{Z} \tau$ be the ring of integers of an imaginary quadratic field $K=\mathbb{Q}(\tau)$; fix the embedding $K \hookrightarrow \mathbb{C}$ so that $\tau \in \mathbb{H}$. Note that $\mathbb{C}$ then becomes a $K$-vector space and in particular an $\mathcal{O}$-module. The choice of $\tau$ as above also determines an embedding

$$
q_{\tau}: K \hookrightarrow M_{2}(\mathbb{Q}) .
$$

The image of its restriction $q_{\tau}: K^{*} \hookrightarrow \mathrm{GL}_{2}^{+}(\mathbb{Q})$ is characterized by the property that (cf. [32] Proposition 4.6)

$$
q_{\tau}\left(K^{*}\right)=\left\{g \in \mathrm{GL}_{2}^{+}(\mathbb{Q}): \quad g(\tau)=\tau\right\} .
$$

For $g=q_{\tau}(x)$ with $x \in K^{*}$, we have $\operatorname{det}(g)=\mathfrak{n}(x)$, where $\mathfrak{n}: K^{*} \rightarrow \mathbb{Q}^{*}$ is the norm map.

Definition 4.1. For $K$ an imaginary quadratic field, a 1-dimensional $K$-lattice $(\Lambda, \phi)$ is a finitely generated $\mathcal{O}$-submodule $\Lambda \subset \mathbb{C}$, such that $\Lambda \otimes_{\mathcal{O}} K \cong K$, together with a morphism of $\mathcal{O}$-modules

$$
\phi: K / \mathcal{O} \rightarrow K \Lambda / \Lambda .
$$

A 1-dimensional $K$-lattice is invertible if $\phi$ is an isomorphism of $\mathcal{O}$-modules.

Notice that in the definition we assume that the $\mathcal{O}$-module structure is compatible with the embeddings of both $\mathcal{O}$ and $\Lambda$ in $\mathbb{C}$.

Lemma 4.2. A 1-dimensional $K$-lattice is, in particular, a 2-dimensional $\mathbb{Q}$-lattice. Moreover, as an $\mathcal{O}$-module, $\Lambda$ is projective.

Proof. First notice that $K \Lambda=\mathbb{Q} \Lambda$, since $\mathbb{Q O}=K$. This, together with $\Lambda \otimes_{\mathcal{O}} K \cong K$, shows that the $\mathbb{Q}$-vector space $\mathbb{Q} \Lambda$ is 2-dimensional. Since $\mathbb{R} \Lambda=\mathbb{C}$, and $\Lambda$ is finitely generated as an abelian group, this shows that $\Lambda$ is a lattice. The basis $\{1, \tau\}$ provides an identification of $K / \mathcal{O}$ with $\mathbb{Q}^{2} / \mathbb{Z}^{2}$, so that we can view $\phi$ as a homomorphism of abelian groups $\phi: \mathbb{Q}^{2} / \mathbb{Z}^{2} \rightarrow \mathbb{Q} \Lambda / \Lambda$. The pair $(\Lambda, \phi)$ thus gives a two dimensional $\mathbb{Q}$-lattice.

As an $\mathcal{O}$-module $\Lambda$ is isomorphic to a finitely generated $\mathcal{O}$-submodule of $K$, hence to an ideal in $\mathcal{O}$. Every ideal in a Dedekind domain $\mathcal{O}$ is finitely generated projective over $\mathcal{O}$.

Suppose given a 1 -dimensional $K$-lattice $(\Lambda, \phi)$. Then the elliptic curve $E=\mathbb{C} / \Lambda$ has complex multiplication by $K$, namely there is an isomorphism

$$
\iota: K \stackrel{\simeq}{\rightarrow} \operatorname{End}(E) \otimes \mathbb{Q} .
$$


In general, for $\Lambda$ a lattice in $\mathbb{C}$, if the elliptic curve $E=\mathbb{C} / \Lambda$ has complex multiplication (i.e. there is an isomorphism (4.8) for $K$ an imaginary quadratic field), then the endomorphisms of $E$ are given by $\operatorname{End}(E)=\mathcal{O}_{\Lambda}$, where $\mathcal{O}_{\Lambda}$ is the order of $\Lambda$,

$$
\mathcal{O}_{\Lambda}=\{x \in K: x \Lambda \subset \Lambda\} .
$$

Notice that the elliptic curves $E=\mathbb{C} / \Lambda$, where $\Lambda$ is a 1-dimensional $K$-lattice, have $\mathcal{O}_{\Lambda}=\mathcal{O}$, the maximal order. The number of distinct isomorphism classes of elliptic curves $E$ with $\operatorname{End}(E)=\mathcal{O}$ is equal to the class number $h_{K}$. All the other elliptic curves with complex multiplication by $K$ are obtained from these by isogenies.

Definition 4.3. Two 1-dimensional $K$-lattices $\left(\Lambda_{1}, \phi_{1}\right)$ and $\left(\Lambda_{2}, \phi_{2}\right)$ are commensurable if $K \Lambda_{1}=$ $K \Lambda_{2}$ and $\phi_{1}=\phi_{2}$ modulo $\Lambda_{1}+\Lambda_{2}$.

One checks as in the case of $\mathbb{Q}$-lattices (cf. [10]) that it is an equivalence relation.

Lemma 4.4. Two 1-dimensional $K$-lattices are commensurable iff the underlying $\mathbb{Q}$-lattices are commensurable. Up to scaling, any $K$-lattice $\Lambda$ is equivalent to a $K$-lattice $\Lambda^{\prime}=\lambda \Lambda \subset K \subset \mathbb{C}$, for a $\lambda \in \mathbb{C}^{*}$. The lattice $\Lambda^{\prime}$ is unique modulo $K^{*}$.

Proof. The first statement holds, since for 1-dimensional $K$-lattices we have $K \Lambda=\mathbb{Q} \Lambda$. For the second statement, the $K$-vector space $K \Lambda$ is 1 -dimensional. If $\xi$ is a generator, then $\xi^{-1} \Lambda \subset K$. The remaining ambiguity is only by scaling by elements in $K^{*}$.

A more explicit description of the space of 1-dimensional $K$-lattices, the commensurability relation, and the action of $\mathbb{C}^{*}$ by scaling is the following (cf. [11]).

Proposition 4.5. The following properties hold.

(1) The data $(\Lambda, \phi)$ of a 1-dimensional $K$-lattice are equivalent to data $(\rho, s)$ of an element $\rho \in \hat{\mathcal{O}}$ and $s \in \mathbb{A}_{K}^{*} / K^{*}$, modulo the $\hat{\mathcal{O}}^{*}$-action given by $(\rho, s) \mapsto\left(x^{-1} \rho, x s\right), x \in \hat{\mathcal{O}}^{*}$. Thus, the space of 1-dimensional $K$-lattices is given by

$$
\hat{\mathcal{O}} \times_{\hat{\mathcal{O}}^{*}}\left(\mathbb{A}_{K}^{*} / K^{*}\right) .
$$

(2) Let $\mathbb{A}_{K}=\mathbb{A}_{K, f} \times \mathbb{C}^{*}$ be the subset of adèles of $K$ with nontrivial archimedean component. The map $\Theta(\rho, s)=\rho s$,

$$
\Theta: \hat{\mathcal{O}} \times_{\hat{\mathcal{O}}^{*}}\left(\mathbb{A}_{K}^{*} / K^{*}\right) \rightarrow \mathbb{A}_{K}^{*} / K^{*}
$$

preserves commensurability and induces an identification of the set of commensurability classes of 1-dimensional $K$-lattices (not up to scale) with the space $\mathbb{A}_{K} / K^{*}$.

(3) The map defined as $\Upsilon:(\Lambda, \phi) \mapsto \rho \in \hat{\mathcal{O}} / K^{*}$ for principal $K$-lattices extends to an identification, given by $\Upsilon:(\Lambda, \phi) \mapsto s_{f} \rho \in \mathbb{A}_{K, f} / K^{*}$, of the set of commensurability classes of 1 -dimensional $K$-lattices up to scaling with the quotient

$$
\hat{\mathcal{O}} / K^{*}=\mathbb{A}_{K, f} / K^{*} \text {. }
$$

The proof ( $c f$. [11]) uses the fact that the $\mathcal{O}$-module $\Lambda$ can be described in the form $\Lambda_{s}=s_{\infty}^{-1}\left(s_{f} \hat{\mathcal{O}} \cap K\right)$, where $s=\left(s_{f}, s_{\infty}\right) \in \mathbb{A}_{K}^{*}$. This satisfies $\Lambda_{k s}=\Lambda_{s}$ for all $k \in\left(\hat{\mathcal{O}}^{*} \times 1\right) K^{*} \subset \mathbb{A}_{K}^{*}$, so that, up to scaling, $\Lambda$ can be identified with an ideal in $\mathcal{O}$, written in the form $s_{f} \hat{\mathcal{O}} \cap K(c f$. [32] §5.2).

The quotient $\mathbb{A}_{K, f} / K^{*}$ has a description in terms of elliptic curves, analogous to what we explained in the case of the $\mathrm{GL}_{2}$-system. In fact, we can associate to $(\Lambda, \phi)$ the data $(E, \eta)$ of an elliptic curve $E=\mathbb{C} / \Lambda$ with complex multiplication (4.8), such that the embedding $K \hookrightarrow \mathbb{C}$ determined by this identification and by the action of $\operatorname{End}(E)$ on the tangent space of $E$ at the origin is the embedding specified by $\tau$ ( $c f .[25]$ p.28, [32] §5.1), and an $\mathbb{A}_{K, f}$-homomorphism

$$
\eta: \mathbb{A}_{K, f} \rightarrow \Lambda \otimes_{\mathcal{O}} \mathbb{A}_{K, f},
$$


The composite map

$$
\mathbb{A}_{K, f} \stackrel{\eta}{\rightarrow} \Lambda \otimes_{\mathcal{O}} \mathbb{A}_{K, f} \stackrel{\simeq}{\rightarrow} \mathbb{A}_{K, f},
$$

determines an element $s_{f} \rho \in \mathbb{A}_{K, f}$. The set of equivalence classes of data $(E, \iota, \eta)$, where equivalence is given by an isogeny of the elliptic curve compatible with the other data, is the quotient $\mathbb{A}_{K, f} / K^{*}$. This generalizes to the non-invertible case the analogous result for invertible 1 -dimensional $K$-lattices (data $(E, \iota, \eta)$, with $\eta$ an isomorphism realized by an element $\left.\rho \in \mathbb{A}_{K, f}^{*}\right)$ treated in $[25]$, where the set of equivalence classes is given by the idèle class group of the imaginary quadratic field,

$$
\mathbb{I}_{K} / K^{*}=\mathrm{GL}_{1}(K) \backslash \mathrm{GL}_{1}\left(\mathbb{A}_{K, f}\right)=C_{K} / D_{K}
$$

\subsection{Algebras of coordinates.}

We now describe the noncommutative algebra of coordinates of the space of commensurability classes of 1 -dimensional $K$-lattices up to scaling.

To this purpose, we first consider the groupoid $\tilde{\mathcal{R}}_{K}$ of the equivalence relation of commensurability on 1-dimensional $K$-lattices (not up to scaling). By construction, this groupoid is a subgroupoid of the groupoid $\tilde{\mathcal{R}}$ of commensurability classes of 2 -dimensional $\mathbb{Q}$-lattices. Its structure as a locally compact étale groupoid is inherited from this embedding.

The groupoid $\tilde{\mathcal{R}}_{K}$ corresponds to the quotient $\mathbb{A}_{K} / K^{*}$. Its $C^{*}$-algebra is given up to Morita equivalence by the crossed product

$$
C_{0}\left(\mathbb{A}_{K}\right) \rtimes K^{*}
$$

The case of commensurability classes of 1 -dimensional $K$-lattices up to scaling is more delicate. In fact, Proposition 4.5 describes set theoretically the space of commensurability classes of 1-dimensional $K$-lattices up to scaling as the quotient $\mathbb{A}_{K, f} / K^{*}$. This has a noncommutative algebra of coordinates, which is the crossed product

$$
C_{0}\left(\mathbb{A}_{K, f} / \mathcal{O}^{*}\right) \rtimes K^{*} / \mathcal{O}^{*}
$$

As we are going to show below, this is Morita equivalent to the noncommutative algebra $\mathcal{A}_{K}=C^{*}\left(G_{K}\right)$ obtained by taking the quotient by scaling $G_{K}=\tilde{\mathcal{R}}_{K} / \mathbb{C}^{*}$ of the groupoid of the equivalence relation of commensurability.

Unlike what happens when taking the quotient by the scaling action of $\mathbb{C}^{*}$ in the $\mathrm{GL}_{2}$-system, in the CM case the quotient $G_{K}=\tilde{\mathcal{R}}_{K} / \mathbb{C}^{*}$ is still a groupoid.

The simplest way to check this is to write $\tilde{\mathcal{R}}_{K}$ as the union of the two groupoids $\tilde{\mathcal{R}}_{K}=G_{0} \cup G_{1}$, corresponding respectively to pairs of commensurable $K$-lattices $\left(L, L^{\prime}\right)$ with $L=(\Lambda, 0), L^{\prime}=\left(\Lambda^{\prime}, 0\right)$ and $\left(L, L^{\prime}\right)$ with $L=(\Lambda, \phi \neq 0), L^{\prime}=\left(\Lambda^{\prime}, \phi^{\prime} \neq 0\right)$. The scaling action of $\mathbb{C}^{*}$ on $G_{0}$ is the identity on $\mathcal{O}^{*}$ and the corresponding action of $\mathbb{C}^{*} / \mathcal{O}^{*}$ is free on the units of $G_{0}$. Thus, the quotient $G_{0} / \mathbb{C}^{*}$ is a groupoid. Similarly, the action of $\mathbb{C}^{*}$ on $G_{1}$ is free on the units of $G_{1}$ and the quotient $G_{1} / \mathbb{C}^{*}$ is a groupoid.

The quotient topology turns $G_{K}$ into a locally compact étale groupoid. The algebra of coordinates $\mathcal{A}_{K}=C^{*}\left(G_{K}\right)$ is described equivalently by restricting the convolution product of the algebra of $\tilde{\mathcal{R}}_{K}$ to weight zero functions with $\mathbb{C}^{*}$-compact support and then passing to the $C^{*}$ completion, as in the $\mathrm{GL}_{2}$-case. In other words the algebra $\mathcal{A}_{K}$ of the $\mathrm{CM}$ system is the convolution algebra of weight zero functions on the groupoid $\tilde{\mathcal{R}}_{K}$ of the equivalence relation of commensurability on $K$ lattices. Elements in the algebra are functions of pairs $(\Lambda, \phi),\left(\Lambda^{\prime}, \phi^{\prime}\right)$ of commensurable 1 -dimensional $K$-lattices satisfying, for all $\lambda \in \mathbb{C}^{*}$,

$$
f\left(\lambda(\Lambda, \phi), \lambda\left(\Lambda^{\prime}, \phi^{\prime}\right)\right)=f\left((\Lambda, \phi),\left(\Lambda^{\prime}, \phi^{\prime}\right)\right) .
$$

Let us compare the setting of (4.16) i.e. the groupoid $\mathbb{A}_{K, f} / \mathcal{O}^{*} \rtimes K^{*} / \mathcal{O}^{*}$ with the groupoid $G_{K}$. In fact, the difference between these two settings can be seen by looking at the case of $K$-lattices with $\phi=0$. In the first case, this corresponds to the point $0 \in \mathbb{A}_{K, f} / \mathcal{O}^{*}$, which has stabilizer $K^{*} / \mathcal{O}^{*}$, hence we obtain the group $C^{*}$-algebra of $K^{*} / \mathcal{O}^{*}$. In the other case, the corresponding groupoid is obtained 
as a quotient by $\mathbb{C}^{*}$ of the groupoid $\tilde{\mathcal{R}}_{K, 0}$ of pairs of commensurable $\mathcal{O}$-modules (finitely generated of rank one) in $\mathbb{C}$. In this case the units of the groupoid $\tilde{\mathcal{R}}_{K, 0} / \mathbb{C}^{*}$ can be identified with the elements of $\mathrm{Cl}(\mathcal{O})$ and the reduced groupoid by any of these units is the group $K^{*} / \mathcal{O}^{*}$. The general result below gives the Morita equivalence in general.

Proposition 4.6. Let $\mathcal{A}_{K, \text { princ }}:=C_{0}\left(\mathbb{A}_{K, f} / \mathcal{O}^{*}\right) \rtimes K^{*} / \mathcal{O}^{*}$. Let $\mathcal{H}$ be the space of pairs up to scaling $\left((\Lambda, \phi),\left(\Lambda^{\prime}, \phi^{\prime}\right)\right)$ of commensurable $K$-lattices, with $(\Lambda, \phi)$ principal. The space $\mathcal{H}^{\prime}$ is defined analogously with $\left(\Lambda^{\prime}, \phi^{\prime}\right)$ principal. Then $\mathcal{H}$ (resp. $\left.\mathcal{H}^{\prime}\right)$ has the structure of $\mathcal{A}_{K \text {,princ }}-\mathcal{A}_{K}$ (resp. $\mathcal{A}_{K^{-}}$ $\left.\mathcal{A}_{K, \text { princ }}\right)$ bimodule. These bimodules give a Morita equivalence between the algebras $\mathcal{A}_{K, \text { princ }}$ and $\mathcal{A}_{K}=C^{*}\left(G_{K}\right)$.

Proof. The correspondence given by these bimodules has the effect of reducing to the principal case. In that case the groupoid of the equivalence relation (not up to scaling) is given by the crossed product $\mathbb{A}_{K} \rtimes K^{*}$. When taking the quotient by $\mathbb{C}^{*}$ we then obtain the groupoid $\mathbb{A}_{K, f} / \mathcal{O}^{*} \rtimes K^{*} / \mathcal{O}^{*}$.

Recall that the $C^{*}$-algebra for the $\mathrm{GL}_{2}$-system is not unital, the reason being that the space of 2dimensional $\mathbb{Q}$-lattices up to scaling is noncompact, due to the presence of the modulus $z \in \mathbb{H}$ of the lattice. When restricting to 1-dimensional $K$-lattices up to scaling, this parameter $z$ affects only finitely many values, corresponding to representatives $\Lambda=\mathbb{Z}+\mathbb{Z} z$ of the classes in $\operatorname{Cl}(\mathcal{O})$.

Thus, the algebra $\mathcal{A}_{K}$ is unital.

In terms of the groupoid, this can be seen from the fact that the set $G_{K}^{(0)}$ of units of $G_{K}$ is the compact space

$$
X=G_{K}^{(0)}=\hat{\mathcal{O}} \times_{\hat{\mathcal{O}}^{*}}\left(\mathbb{A}_{K, f}^{*} / K^{*}\right) .
$$

obtained as the quotient of $\hat{\mathcal{O}} \times_{\hat{\mathcal{O}}^{*}}\left(\mathbb{A}_{K}^{*} / K^{*}\right)$ by the action of $\mathbb{C}^{*}$. Notice that $\mathbb{A}_{K, f}^{*} /\left(K^{*} \times \hat{\mathcal{O}}^{*}\right)$ is $\mathrm{Cl}(\mathcal{O})$.

By restriction from the $\mathrm{GL}_{2}$-system, there is a homomorphism $\mathfrak{n}$ from the groupoid $\tilde{\mathcal{R}}_{K}$ to $\mathbb{R}_{+}^{*}$ given by the covolume of a commensurable pair of $K$-lattices. More precisely given such a pair $\left(L, L^{\prime}\right)=$ $\left((\Lambda, \phi),\left(\Lambda^{\prime}, \phi^{\prime}\right)\right)$ we let

$$
\left|L / L^{\prime}\right|=\text { covolume }\left(\Lambda^{\prime}\right) / \operatorname{covolume}(\Lambda)
$$

This is invariant under scaling both lattices, so it is defined on $G_{K}=\tilde{\mathcal{R}}_{K} / \mathbb{C}^{*}$. Up to scale, we can identify the lattices in a commensurable pair with ideals in $\mathcal{O}$. The covolume is then given by the ratio of the norms. This defines a time evolution on the algebra $\mathcal{A}_{K}$ by

$$
\sigma_{t}(f)\left(L, L^{\prime}\right)=\left|L / L^{\prime}\right|^{i t} f\left(L, L^{\prime}\right) .
$$

We construct representations for the algebra $\mathcal{A}_{K}$. For an étale groupoid $G_{K}$, every unit $y \in G_{K}^{(0)}$ defines a representation $\pi_{y}$ by left convolution of the algebra of $G_{K}$ in the Hilbert space $\mathcal{H}_{y}=$ $\ell^{2}\left(\left(G_{K}\right)_{y}\right)$, where $\left(G_{K}\right)_{y}$ is the set of elements with source $y$. The representations corresponding to points that have a nontrivial automorphism group will no longer be irreducible. As in the $\mathrm{GL}_{2}$-case, this defines the norm on $\mathcal{A}_{K}$ as

$$
\|f\|=\sup _{y}\left\|\pi_{y}(f)\right\| .
$$

Notice that, unlike in the case of the $\mathrm{GL}_{2}$-system, we are dealing here with amenable groupoids, hence the distinction between the maximal and the reduced $C^{*}$-algebra does not arise.

The following result of [11] shows the relation to the Dedekind zeta function.

Lemma 4.7. (1) Given an invertible K-lattice $(\Lambda, \phi)$, the map

$$
\left(\Lambda^{\prime}, \phi^{\prime}\right) \mapsto J=\left\{x \in \mathcal{O} \mid x \Lambda^{\prime} \subset \Lambda\right\}
$$

gives a bijection of the set of $K$-lattices commensurable to $(\Lambda, \phi)$ with the set of ideals in $\mathcal{O}$.

(2) Invertible $K$-lattices define positive energy representations. 
(3) The partition function is the Dedekind zeta function $\zeta_{K}(\beta)$ of $K$.

As in Theorem 1.26 and Lemma 1.27 of [10] in the $\mathrm{GL}_{2}$-case, one uses the fact that, if $\Lambda$ is an invertible 2 -dimensional $\mathbb{Q}$-lattice and $\Lambda^{\prime}$ is commensurable to $\Lambda$, then $\Lambda \subset \Lambda^{\prime}$. The map above is well defined, since $J \subset \mathcal{O}$ is an ideal. Moreover, $J \Lambda^{\prime}=\Lambda$, since $\mathcal{O}$ is a Dedekind domain. It is shown in [11] that the map is both injective and surjective. We use the notation

$$
J^{-1}(\Lambda, \phi)=\left(\Lambda^{\prime}, \phi\right) .
$$

For an invertible $K$-lattice, this gives an identification of $\left(G_{K}\right)_{y}$ with the set $\mathcal{J}$ of ideals $J \subset \mathcal{O}$. The covolume is then given by the norm. The corresponding Hamiltonian is of the form

$$
H \epsilon_{J}=\log \mathfrak{n}(J) \epsilon_{J},
$$

with non-negative eigenvalues, hence the partition function is the Dedekind zeta function

$$
Z(\beta)=\sum_{J \text { ideal in } \mathcal{O}} \mathfrak{n}(J)^{-\beta}=\zeta_{K}(\beta) .
$$

\subsection{Symmetries.}

We shall now adapt the discussion of symmetries of the GL(2) system to $K$-lattices, adopting a contravariant notation instead of the covariant one used in [10].

Proposition 4.8. The semigroup $\hat{\mathcal{O}} \cap \mathbb{A}_{K, f}^{*}$ acts on the algebra $\mathcal{A}_{K}$ by endomorphisms. The subgroup $\hat{\mathcal{O}}^{*}$ acts on $\mathcal{A}_{K}$ by automorphisms. The subsemigroup $\mathcal{O}^{\times}$acts by inner endomorphisms.

Consider the set of $K$-lattices $(\Lambda, \phi)$ such that $\phi$ is well defined modulo $J \Lambda$, for an ideal $J=s \hat{\mathcal{O}} \cap K$. Namely, the map $\phi: K / \mathcal{O} \longrightarrow K \Lambda / \Lambda$ factorises as $K / \mathcal{O} \longrightarrow K \Lambda / J \Lambda \rightarrow K \Lambda / \Lambda$. We say, in this case, that the $K$-lattice $(\Lambda, \phi)$ is divisible by $J$. For a commensurable pair $(\Lambda, \phi)$ and $\left(\Lambda^{\prime}, \phi^{\prime}\right)$, and an element $f \in \mathcal{A}_{K}$, we set

$$
\theta_{s}(f)\left((\Lambda, \phi),\left(\Lambda^{\prime}, \phi^{\prime}\right)\right)= \begin{cases}f\left(\left(\Lambda, s^{-1} \phi\right),\left(\Lambda^{\prime}, s^{-1} \phi^{\prime}\right)\right) & \text { both } K \text {-lattices are divisible by } J \\ 0 & \text { otherwise. }\end{cases}
$$

Formula (4.25) defines an endomorphism of $\mathcal{A}_{K}$ with range the algebra reduced by an idempotent $e_{J}$. Clearly, for $s \in \hat{\mathcal{O}}^{*}$, the above defines an automorphism, which is compatible with the time evolution. For $s \in \mathcal{O}^{\times}$, the endomorphism (4.25) is inner,

$$
\theta_{s}(f)=\mu_{s} f \mu_{s}^{*}
$$

with $\mu_{s} \in \mathcal{A}_{K}$ given by

$$
\mu_{s}\left((\Lambda, \phi),\left(\Lambda^{\prime}, \phi^{\prime}\right)\right)=\left\{\begin{array}{cc}
1 & \Lambda=s^{-1} \Lambda^{\prime} \text { and } \phi^{\prime}=\phi \\
0 & \text { otherwise. }
\end{array}\right.
$$

The range of $\mu_{s}$ is the projection $e_{J}$, with $J$ the principal ideal generated by $s$. The isometries $\mu_{s}$ are eigenvectors of the time evolution, namely $\sigma_{t}\left(\mu_{s}\right)=\mathfrak{n}(s)^{i t} \mu_{s}$.

Recall that $\mathbb{A}_{K, f}=\hat{\mathcal{O}} \cdot K^{*}$. Thus, we can pass to the corresponding group of symmetries, modulo inner, which is given by the idèle class group $\mathbb{A}_{K, f}^{*} / K^{*}$, which is identified, by the class field theory isomorphism, with the Galois group $\operatorname{Gal}\left(K^{a b} / K\right)$.

This shows that we have an action of the idèle class group on the set of extremal $\mathrm{KMS}_{\beta}$ states of the CM system. The action of the subgroup $\hat{\mathcal{O}}^{*} / \mathcal{O}^{*}$ is by automorphisms, while the action of the quotient group $\mathrm{Cl}(\mathcal{O})$ is by endomorphisms, as we expected according to diagram (4.4). 


\subsection{Comparison with other systems.}

First we investigate the functoriality issue for the CM-system associated to a quadratic imaginary extension $K$ of $\mathbb{Q}$ as above. This describes the compatibility between the CM system and the BC system.

By Proposition 4.6 we can replace the $C^{*}$-algebra $\mathcal{A}_{K}$ by the Morita equivalent one $\mathcal{A}_{K, \text { princ }}=$ $C_{0}\left(\mathbb{A}_{K, f} / \mathcal{O}^{*}\right) \rtimes K^{*} / \mathcal{O}^{*}$. We need to relate it to the BC-system i.e. again, up to Morita equivalence, to the crossed product algebra $C_{0}\left(\mathbb{A}_{f}\right) \rtimes \mathrm{GL}_{1}(\mathbb{Q})^{+}$.

Let $N: \mathbb{A}_{K, f} \rightarrow \mathbb{A}_{f}$ be the norm map and $\iota: N\left(K^{*}\right) \rightarrow K^{*}$ be a group homomorphism section of $N: K^{*} \rightarrow N\left(K^{*}\right)$.

Proposition 4.9. The pair $(N, \iota)$ induces a morphism

$$
\varrho: C_{0}\left(\mathbb{A}_{f}\right) \rtimes N\left(K^{*}\right) \rightarrow M\left(C_{0}\left(\mathbb{A}_{K, f} / \mathcal{O}^{*}\right) \rtimes K^{*} / \mathcal{O}^{*}\right)=M\left(\mathcal{A}_{K, \text { princ }}\right)
$$

to the multiplier algebra of $\mathcal{A}_{K, \text { princ }}$.

Proof. This is an immediate application of the discussion in Section 3.7. In fact, one checks that condition (3.62) is fulfilled by construction. Note that, in the setup as above, $N\left(K^{*}\right)$ is a subgroup of $\mathrm{GL}_{1}(\mathbb{Q})^{+}$and, moreover, $N\left(\mathcal{O}^{*}\right)=1$. The noncommutative space $\mathbb{A}_{f} / N\left(K^{*}\right)$, quotient of $\mathbb{A}_{f}$ by $N\left(K^{*}\right)$, is an etale infinite covering of the space of one dimensional $\mathbb{Q}$-lattices up to scaling.

It is also useful to see explicitly the relation of the algebra $\mathcal{A}_{K}$ of the CM system to the algebras previously considered in generalizations of the Bost-Connes results, especially those of [5], [16], and [20]. This will explain why the algebra $\mathcal{A}_{K}$ contains exactly the amount of extra information to allow for the full explict class field theory to appear.

The partition function of the system considered in [16] agrees with the Dedekind zeta function only in the case of class number one. A different system, which has partition function the Dedekind zeta function in all cases, was introduced in [5]. Our system also has as partition function the Dedekind zeta function, independently of class number. It however differs from the system of [5]. In fact, in the latter, which is a semigroup crossed product, the natural quotient of the $C^{*}$-algebra obtained by specializing at the fixed point of the semigroup is the group ring of an extension of the class group $\mathrm{Cl}(\mathcal{O})$ by $K^{*} / \mathcal{O}^{*}$, while in our case, when specializing similarly to the $K$-lattices with $\phi=0$, we obtain an algebra Morita equivalent to the group ring of $K^{*} / \mathcal{O}^{*}$. Thus, the two systems are not naturally Morita equivalent.

The system considered in [20] is analyzed there only under the hypothesis of class number one. It can be recovered from our system, which has no restrictions on class number, by reduction to those $K$ lattices that are principal. Thus, the system of [20] is Morita equivalent to our system (cf. Proposition 4.6).

Notice, moreover, that the crossed product algebra $C(\hat{\mathcal{O}}) \rtimes \mathcal{O}^{\times}$considered in some generalizations of the BC system is more similar to the "determinant part" of the $\mathrm{GL}_{2}$-system ( $c f$. Section 1.7 of [10]), namely to the algebra $C\left(M_{2}(\hat{\mathbb{Z}})\right) \rtimes M_{2}^{+}(\mathbb{Z})$, than to the full $\mathrm{GL}_{2}$-system.

\section{KMS STATES AND COMPLEX MULTIPLICATION}

We can now describe the arithmetic subalgebra $\mathcal{A}_{K, \mathbb{Q}}$ of $\mathcal{A}_{K}$. The relation between the CM and the $\mathrm{GL}_{2}$-system provides us with a natural choice for $\mathcal{A}_{K, \mathbb{Q}}$.

Definition 5.1. The algebra $\mathcal{A}_{K, \mathbb{Q}}$ is the $K$-algebra obtained by

$$
\mathcal{A}_{K, \mathbb{Q}}=\left.\mathcal{A}_{2, \mathbb{Q}}\right|_{G_{K}} \otimes_{\mathbb{Q}} K \text {. }
$$

Here $\left.\mathcal{A}_{2, \mathbb{Q}}\right|_{G_{K}}$ denotes the $\mathbb{Q}$-algebra obtained by restricting elements of the algebra $\mathcal{A}_{2, \mathbb{Q}}$ of Definition 3.8 to the $\mathbb{C}^{*}$-quotient $G_{K}$ of the subgroupoid $\tilde{\mathcal{R}}_{K} \subset \tilde{\mathcal{R}}_{2}$.

Notice that, for the CM system, $\mathcal{A}_{K, \mathbb{Q}}$ is a subalgebra of $\mathcal{A}_{K}$, not just a subalgebra of unbounded multipliers as in the $\mathrm{GL}_{2}$-system, because of the fact that $\mathcal{A}_{K}$ is unital. 
We are now ready to state the main result of the $\mathrm{CM}$ case. The following theorem gives the structure of KMS states for the system $\left(\mathcal{A}_{K}, \sigma_{t}\right)$ and shows that this system gives a solution to Problem 1.1 for the imaginary quadratic field $K$.

Theorem 5.2. (Connes-Marcolli-Ramachandran [11]) Consider the system $\left(\mathcal{A}_{K}, \sigma_{t}\right)$ described in the previous section. The extremal KMS states of this system satisfy:

- In the range $0<\beta \leq 1$ there is a unique KMS state.

- For $\beta>1$, extremal $K M S_{\beta}$ states are parameterized by invertible $K$-lattices,

$$
\mathcal{E}_{\beta} \simeq \mathbb{A}_{K, f}^{*} / K^{*}
$$

with a free and transitive action of $C_{K} / D_{K} \cong \mathbb{A}_{K, f}^{*} / K^{*}$ as symmetries.

- In this range, the extremal $K M S_{\beta}$ state associated to an invertible $K$-lattice $L=(\Lambda, \phi)$ is of the form

$$
\varphi_{\beta, L}(f)=\zeta_{K}(\beta)^{-1} \sum_{J \text { ideal in } \mathcal{O}} f\left(J^{-1} L, J^{-1} L\right) \mathfrak{n}(J)^{-\beta},
$$

where $\zeta_{K}(\beta)$ is the Dedekind zeta function, and $J^{-1} L$ defined as in (4.22).

- The set of extremal $K M S_{\infty}$ states (as weak limits of $K M S_{\beta}$ states) is still given by (5.2).

- The extremal $K M S_{\infty}$ states $\varphi_{\infty, L}$ of the CM system, evaluated on the arithmetic subalgebra $\mathcal{A}_{K, \mathbb{Q}}$, take values in $K^{a b}$, with $\varphi_{\infty, L}\left(\mathcal{A}_{K, \mathbb{Q}}\right)=K^{a b}$.

- The class field theory isomorphism (1.2) intertwines the action of $\mathbb{A}_{K, f}^{*} / K^{*}$ by symmetries of the system $\left(\mathcal{A}_{K}, \sigma_{t}\right)$ and the action of $\operatorname{Gal}\left(K^{a b} / K\right)$ on the image of $\mathcal{A}_{K, \mathbb{Q}}$ under the extremal $K M S_{\infty}$ states. Namely, for all $\varphi_{\infty, L} \in \mathcal{E}_{\infty}$ and for all $f \in \mathcal{A}_{K, \mathbb{Q}}$,

$$
\alpha\left(\varphi_{\infty, L}(f)\right)=\left(\varphi_{\infty, L} \circ \theta^{-1}(\alpha)\right)(f), \quad \forall \alpha \in \operatorname{Gal}\left(K^{a b} / K\right) .
$$

Notice that the result stated above is substantially different from the $\mathrm{GL}_{2}$-system. This is not surprising, as the following general fact illustrates. Given an étale groupoid $\mathcal{G}$ and a full subgroupoid $\mathcal{G}^{\prime} \subset \mathcal{G}$, let $\rho$ be a homomorphism $\rho: \mathcal{G} \rightarrow \mathbb{R}_{+}^{*}$. The inclusion $\mathcal{G}^{\prime} \subset \mathcal{G}$ gives a correspondence between the $C^{*}$-algebras associated to $\mathcal{G}^{\prime}$ and $\mathcal{G}$, compatible with the time evolution associated to $\rho$ and its restriction to $\mathcal{G}^{\prime}$. The following simple example, however, shows that, in general, the KMS states for the $\mathcal{G}^{\prime}$ system do not map to KMS states for the $\mathcal{G}$ system. We let $\mathcal{G}$ be the groupoid with units $\mathcal{G}^{(0)}$ given by an infinite countable set, and morphisms given by all pairs of units. Consider a finite subset of $\mathcal{G}^{(0)}$ and let $\mathcal{G}^{\prime}$ be the reduced groupoid. Finally, let $\rho$ be trivial. Clearly, the $\mathcal{G}^{\prime}$ system admits a KMS state for all temperatures given by the trace, while, since there is no tracial state on the compact operators, the $\mathcal{G}$ system has no KMS states.

\subsection{Low temperature KMS states and Galois action.}

The partition function $Z_{K}(\beta)$ of (4.24) converges for $\beta>1$. We have also seen in the previous section that invertible $K$-lattices $L=(\Lambda, \phi)$ determine positive energy representations of $\mathcal{A}_{K}$ on the Hilbert space $\mathcal{H}=\ell^{2}(\mathcal{J})$ where $\mathcal{J}$ is the set of ideals of $\mathcal{O}$. Thus, the formula

$$
\varphi_{\beta, L}(f)=\frac{\operatorname{Tr}\left(\pi_{L}(f) \exp (-\beta H)\right)}{\operatorname{Tr}(\exp (-\beta H))}
$$

defines an extremal $\mathrm{KMS}_{\beta}$ state, with the Hamiltonian $H$ of (4.23). These states are of the form (5.3). It is not hard to see that distinct elements in $\mathbb{A}_{K, f} / K^{*}$ define distinct states $\varphi_{\beta, L}$. This shows that we have an injection of $\mathbb{A}_{K, f} / K^{*} \subset \mathcal{E}_{\beta}$. Conversely, every extremal $\mathrm{KMS}_{\beta}$ state is of the form (5.3). This is shown in [11] by first proving that $\mathrm{KMS}_{\beta}$ states are given by measures on the space $X$ of $K$-lattices (up to scaling),

$$
\varphi(f)=\int_{X} f(L, L) d \mu(L), \quad \forall f \in \mathcal{A}_{K} .
$$

One then shows that, when $\beta>1$ this measure is carried by the commensurability classes of invertible $K$-lattices. (We refer the reader to [11] for details.) 
The weak limits as $\beta \rightarrow \infty$ of states in $\mathcal{E}_{\beta}$ define states in $\mathcal{E}_{\infty}$ of the form

$$
\varphi_{\infty, L}(f)=f(L, L) .
$$

Some care is needed in defining the action of the symmetry group $\mathbb{A}_{K, f} / K^{*}$ on extremal states at zero temperature. In fact, as it happens also in the $\mathrm{GL}_{2}$-case, for an invertible $K$-lattice evaluating $\varphi_{\infty, L}$ on $\theta_{s}(f)$ does not give a nontrivial action in the case of endomorphisms. However, there is a nontrivial action induced on $\mathcal{E}_{\infty}$ by the action on $\mathcal{E}_{\beta}$ for finite $\beta$ and it is obtained as

$$
\Theta_{s}\left(\varphi_{\infty, L}\right)(f)=\lim _{\beta \rightarrow \infty}\left(W_{\beta}\left(\varphi_{\infty, L}\right) \circ \theta_{s}\right)(f),
$$

where $W_{\beta}$ is the "warm up" map (2.7). This gives

$$
\Theta_{s}\left(\varphi_{\infty, L}\right)=\varphi_{\infty, L_{s}},
$$

with $L_{s}$ the invertible $K$-lattice $\left(J_{s}^{-1} \Lambda, s^{-1} \phi\right)$.

Thus the action of the symmetry group $\mathbb{I}_{K} / K^{*}$ is given by

$$
L=(\Lambda, \phi) \mapsto L_{s}=\left(J_{s}^{-1} \Lambda, s^{-1} \phi\right), \quad \forall s \in \mathbb{I}_{K} / K^{*} .
$$

When we evaluate states $\varphi_{\infty, L}$ on elements $f \in \mathcal{A}_{K, \mathbb{Q}}$ of the arithmetic subalgebra we obtain

$$
\varphi_{\infty, L}(f)=f(L, L)=g(L),
$$

where the function $g$ is the lattice function of weight 0 obtained as the restriction of $f$ to the diagonal. By construction of $\mathcal{A}_{K, \mathbb{Q}}$, one obtains in this way all the evaluations $f \mapsto f(z)$ of elements of the modular field $F$ on the finitely many modules $z \in \mathbb{H}$ of the classes of $K$-lattices.

The modular functions $f \in F$ that are defined at $\tau$ define a subring $B$ of $F$. The theory of complex multiplication ( $c f .[32], \S 5)$ shows that the subfield $F_{\tau} \subset \mathbb{C}$ generated by the values $f(\tau)$, for $f \in B$, is the maximal abelian extension of $K$ (we have fixed an embedding $K \subset \mathbb{C}$ ),

$$
F_{\tau}=K^{a b} .
$$

Moreover, the action of $\alpha \in \operatorname{Gal}\left(K^{a b} / K\right)$ on the values $f(z)$ is given by

$$
\alpha f(z)=f^{\sigma q_{\tau} \theta^{-1}(\alpha)}(z) .
$$

In this formula the notation $f \mapsto f^{\gamma}$ denotes the action of an element $\gamma \in \operatorname{Aut}(F)$ on the elements $f \in F$, the map $\theta$ is the class field theory isomorphism (1.2), $q_{\tau}$ is the embedding of $\mathbb{A}_{K, f}^{*}$ in $\mathrm{GL}_{2}(\mathbb{A})$ and $\sigma$ is as in the diagram with exact rows

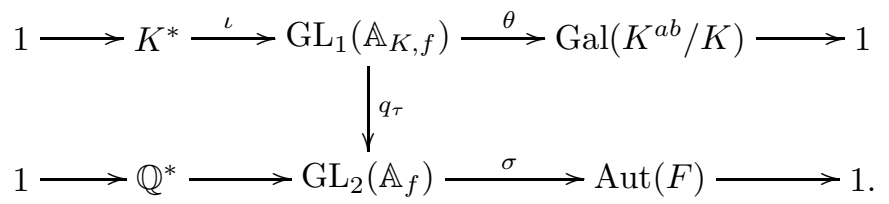

Thus, when we act by an element $\alpha \in \operatorname{Gal}\left(K^{a b} / K\right)$ on the values on $\mathcal{A}_{K, \mathbb{Q}}$ of an extremal $\mathrm{KMS}_{\infty}$ state we have

$$
\alpha \varphi_{\infty, L}(f)=\varphi_{\infty, L_{s}}(f)
$$

where $s=\theta^{-1}(\alpha) \in \mathbb{I}_{K} / K^{*}$.

This corresponds to the result of Theorem 1.39 of [10] for the case of 2-dimensional $\mathbb{Q}$-lattices (see equations (1.130) and following in [10]) with the slight nuance that we used there a covariant notation for the Galois action rather than the traditional contravariant one $f \mapsto f^{\gamma}$. 


\subsection{Open Questions.}

Theorem 5.2 shows the existence of a $C^{*}$-dynamical system $\left(\mathcal{A}_{K}, \sigma_{t}\right)$ with all the required properties for the interpretation of the class field theory isomorphism in the CM case in the framework of fabulous states. There is however still one key feature of the BC-system that needs to be obtained in this framework. It is the presentation of the arithmetic subalgebra $\mathcal{A}_{K, \mathbb{Q}}$ in terms of generators and relations. This should be obtained along the lines of [10] Section 6, Lemma 15 and Proposition 15, and Section 9 Proposition 41. These suggest that the relations will have coefficients in the Hilbert modular field.

We only handled in this paper the CM-case i.e. imaginary quadratic fields, but many of the notions we introduced such as that of a $K$-lattice should be extended to arbitrary number fields $K$. Note in that respect that Proposition 4.5 indicates clearly that, in general, the space of commensurability classes of $K$-lattices should be identical to the space $\mathbb{A}_{K} / K^{*}$ of Adèle classes introduced in [8] for the spectral realization of zeros of $L$-functions, with the slight nuance of non-zero archimedan component. The scaling group which is used to pass from the above "dual system" to the analogue of the BC system is given in the case $K=\mathbb{Q}$ by the group $\mathbb{R}_{+}^{*}$ and in the case of imaginary quadratic fields by the multiplicative group $\mathbb{C}^{*}$. It is thus natural to expect in general that it will be given by the connected component of identity $D_{K}$ in the group $C_{K}$ of idèle classes. A possible construction in terms of $K$-lattices should be compared with the systems for number fields recently constructed in [13].

There is another important conceptual point that deserves further investigation. In the classical classfield theory and Langlands program for a field $K$, the main object which is constructed is a certain "space", which is acted upon by two mutually commuting sets of operators: one is the Galois group of an extension of $K$, while the other is a group (algebra, semigroup, set) of geometric symmetries (like Hecke operators). In the very special case of abelian class field theory, it turns out that the commutator of each of these sets essentially coincides with it (or with an appropriate completion), so that these two groups can be naturally identified. The quantum statistical mechanical systems considered in this paper, as well as the mentioned generalizations, give new geometric objects, in the form of quantum statistical mechanical systems, supporting a similar type of geometric symmetries (e.g. groups of symmetries of adelic nature associated to Shimura varieties). The intertwining between geometric and Galois actions is provided by the extremal KMS states at zero temperature and by the presence of an arithmetic subalgebra. Even in the abelian cases, like the BC system and the CM system, it is important to maintain the conceptual difference between geometric and Galois actions. For instance, in the CM case the intertwining via the states, which provides the identification, relies essentially on the classical theory of complex multiplication, through the use of Shimura reciprocity. In the non-abelian setting, this distinction becomes essential. One can already see that clearly in the case of the $\mathrm{GL}_{2}$-system, where for a generic set of extremal KMS states at zero temperature one still obtains a Galois interpretation of the geometric action (again by means of the classical theory of Shimura reciprocity and automorphisms of the modular field), while other phenomena appear for non-generic states, which reflect more clearly the difference between these two actions. For more general case associated to Shimura varieties, while the geometric action occurs naturally as in the $\mathrm{GL}_{2}$-case and the number fields case, the Galois side is still not understood.

\section{REFERENCES}

[1] J. Arledge, M. Laca, I. Raeburn, Semigroup crossed products and Hecke algebras arising from number fields, Doc. Math. 2 (1997), 115-138 (electronic).

[2] J.-B. Bost and A. Connes, Produits Euleriens et facteurs de type III, C. R. Acad. Sci. Paris Sér. I Math. Vol.315 (1992), no. 3, 279-284.

[3] J.B. Bost, A. Connes, Hecke algebras, Type III factors and phase transitions with spontaneous symmetry breaking in number theory, Selecta Math. (New Series) Vol.1 (1995) N.3, 411-457.

[4] O. Bratteli, D.W. Robinson, Operator algebras and quantum statistical mechanics I,II, Springer Verlag, 1981.

[5] P.B. Cohen, $A C^{*}$-dynamical system with Dedekind zeta partition function and spontaneous symmetry breaking, Journal de Théorie des Nombres de Bordeaux 11 (1999) 15-30. 
[6] A. Connes, Une classification des facteurs de type III, Ann. Sci. École Norm. Sup. (4) 6 (1973), 133-252.

[7] A. Connes, $C^{*}$ algèbres et géométrie différentielle. C. R. Acad. Sci. Paris Sér. A-B 290 (1980), no. 13, A599-A604.

[8] A. Connes, Trace formula in noncommutative geometry and the zeros of the Riemann zeta function, Selecta Math. (New Series) Vol.5 (1999) 29-106.

[9] A. Connes, M. Douglas, A. Schwarz, Noncommutative geometry and Matrix theory: compactification on tori. J. High Energy Phys. (1998) no. 2, Paper 3, 35 pp. (electronic)

[10] A. Connes, M. Marcolli, From physics to number theory via noncommutative geometry. Part I: Quantum statistical mechanics of $\mathbb{Q}$-lattices, preprint 2004.

[11] A. Connes, M. Marcolli, N. Ramachandran KMS states and complex multiplication, to appear in Selecta Mathematica.

[12] A. Connes, H. Moscovici, Modular Hecke algebras and their Hopf symmetry, preprint math.QA/0301089.

[13] E. Ha, F. Paugam, Bost-Connes-Marcolli systems for Shimura varieties. I. Definitions and formal analytic properties, preprint math.OA/0507101 (to appear in IMRN)

[14] R. Haag, Local Quantum Physics, Springer, Berlin 1992.

[15] R. Haag, N. M. Hugenholtz, M. Winnink On the equilibrium states in quantum statistical mechanics, Comm. Math. Phys. 5 (1967), 215, 236.

[16] D. Harari, E. Leichtnam, Extension du phénomène de brisure spontanée de symétrie de Bost-Connes au cas des corps globaux quelconques, Selecta Math. (New Ser.) 3 (1997) 205-243.

[17] D. Kubert, S. Lang, Modular units, Springer 1981.

[18] M. Laca, Semigroups of *-endomorphisms, Dirichlet series, and phase transitions, J. Funct. Anal. 152 (1998) $330-378$.

[19] M. Laca, From endomorphisms to automorphisms and back: dilations and full corners, J. London Math. Soc. (2) 61 (2000) 893-904.

[20] M. Laca, M. van Frankenhuijsen, Phase transitions on Hecke $C^{*}$-algebras and class-field theory over $\mathbb{Q}$, preprint math.OA/0410305.

[21] S. Lang, Elliptic functions, (Second Edition), Graduate Texts in Mathematics 112, Springer-Verlag 1987.

[22] Yu.I. Manin, Real Multiplication and noncommutative geometry, preprint arXiv math.AG/0202109.

[23] Yu.I. Manin, Von Zahlen und Figuren, preprint arXiv math.AG/0201005.

[24] Yu.I. Manin, M. Marcolli, Continued fractions, modular symbols, and noncommutative geometry, Selecta Math. (New Series) Vol.8 (2002) N.3, 475-520.

[25] J.S. Milne, Canonical models of Shimura curves, manuscript, 2003 (www.jmilne.org).

[26] J. Milne, Fields and Galois theory, manuscript, (www.jmilne.org).

[27] J. Milnor, Introduction to algebraic K-theory, Ann. Math. Studies, Vol.72, Princeton University Press, 1971.

[28] S. Neshveyev, Ergodicity of the action of the positive rationals on the group of finite adeles and the Bost-Connes phase transition theorem, Proc. Amer. Math. Soc. 130 (2002), no. 10, 2999-3003.

[29] F. Paugam, Three examples of noncommutative boundaries of Shimura varieties, preprint math.AG/0410254.

[30] W. Rudin, Real and Complex Analysis, McGraw-Hill, NewYork 1987.

[31] B. Schoeneberg, Elliptic Modular Functions, Springer, 1974.

[32] G. Shimura, Arithmetic theory of automorphic functions, Iwanami Shoten and Princeton 1971.

[33] P. Stevenhagen, Hilbert's 12th problem, complex multiplication and Shimura reciprocity, Advanced Studies in Pure Math. 30 (2001) "Class Field Theory - its centenary and prospect" pp. 161-176.

[34] A. Weil, Elliptic functions according to Eisenstein and Kronecker, Springer 1976.

A. Connes: Collège de France, 3, Rue D'Ulm, Paris, F-75005 France

E-mail address: alain@connes.org

M. Marcolli: Max-Planck Institut für Mathematik, Vivatsgasse 7, Bonn, D-53111 Germany

E-mail address: marcolli@mpim-bonn.mpg.de

N. Ramachandran: Department of Mathematics, University of Maryland, College Park, MD 20912 USA

E-mail address: atma@math.umd.edu 